\title{
Diagnosis of the Dynamic Efficiency of Latent Heat Release and the Rapid Intensification of Supertyphoon Haiyan (2013)
}

\author{
Hung-Chi Kuo, Satoki Tsujino, and Chien-Chang Huang \\ Department of Atmospheric Sciences, National Taiwan University, Taipei, Taiwan \\ CHUNG-CHIEH WANG \\ Department of Earth Sciences, National Taiwan Normal University, Taipei, Taiwan \\ KAZUHISA TSUBOKI \\ Institute for Space-Earth Environmental Research, Nagoya University, Nagoya, Japan
}

(Manuscript received 25 April 2018, in final form 2 January 2019)

\begin{abstract}
Before landfall in the Philippines, Supertyphoon Haiyan in 2013 experienced a period of rapid intensification (RI) with the maximum wind speed increasing by $31 \mathrm{~m} \mathrm{~s}^{-1}$ in $24 \mathrm{~h}$. A 2-km horizontal resolution full-physics cloud-resolving model is used to study the RI of Haiyan. To aid in the diagnosis of RI, we employ a dynamic efficiency factor (DEF), which is based on axisymmetric gradient wind vortex theory. The DEF measures the efficacy of convective heating at any point in generating kinetic energy. The DEF is large in the presence of strong baroclinicity near the eyewall. The simulated tropical cyclone (TC) becomes more symmetric and resembles the observations of Haiyan during the RI period. Our results highlight the nonlinear feedback process in which deep convection becomes collocated with the large DEF area near the eyewall. With the intensity increase, the eyewall convection results in significant subsidence warming at the edge of the eye rather than at the center of the eye. The overall eye warming enhances the baroclinicity near the eyewall and the system DEF in the eye-core region is increased by $33 \%$ before and after the RI onset. The vortex intensity increase during the RI period also leads to large unbalanced radial inflow in the boundary layer. The large radial inflow causes the deep convection to occur in the region of large inertial stability inside the radius of maximum wind. The deep convection further efficiently intensifies the TC. Our simulation emphasizes the importance of model resolution in simulating the internal nonlinear processes of RI.
\end{abstract}

\section{Introduction}

While forecast skill of tropical cyclone (TC) tracks have steadily improved during the past decades, forecasts of intensity have made much slower progress and remain a highly challenging problem (Elsberry et al. 2007; DeMaria et al. 2007, 2014; Rogers et al. 2013a). Large intensity errors tend to occur for TCs that undergo rapid intensification (RI; Ito 2016), which is usually defined as a process where the maximum wind speed $V_{\max }$ increases at the 95th percentile of all TC intensity changes over water (Kaplan and DeMaria 2003), or greater than $19.5 \mathrm{~m} \mathrm{~s}^{-1}$ in $24 \mathrm{~h}$ in the western North Pacific (WNP; Hendricks et al. 2010).

Based on the statistical study of Shu et al. (2012), nearly $70 \%$ of all TCs in the 1970-2007 period that

Corresponding author: Chung-Chieh Wang, cwang@ntnu.edu.tw reached $51 \mathrm{~m} \mathrm{~s}^{-1}$ in $V_{\max }$ ( $\geq$ category 3 on the SaffirSimpson hurricane wind scale), experienced RI at least once during their lifetime. While this percentage is much lower for less intense TCs (e.g., 30\% for cases with $41.5 \leq V_{\max } \leq 50.9 \mathrm{~m} \mathrm{~s}^{-1}$, and about $16 \%$ among all TCs), RI is a frequent path for TCs to reach the higher portion of the intensity spectrum. Conversely, the remaining $30 \%$ of severe TCs reached their peak intensity gradually without RI taking place.

Kaplan and DeMaria (2003) identified synoptic conditions conducive to RI, including weaker vertical wind shear, an upper-level trough, a cold-core low, high relative humidity at low-levels, warmer SST, and stronger upper-level easterlies. Hendricks et al. (2010) studied the RI environmental conditions of TCs in the WNP and in the Atlantic during 2003-08 according to the 24-h intensity changes in $V_{\text {max }}$. Their results for the WNP 
indicate no significant difference between the intensifying group and the RI group in most of the environmental conditions such as deep-layer shear, humidity, low-level vorticity and convergence, and upper-level divergence. In other words, once a favorable environment exists for the TCs to intensify, there is no further distinction in synoptic conditions on whether an RI will occur or not.

Statistical studies based on satellite data, however, have found some differences in inner-core rainfall structure between RI and non-RI storms. The RI group tends to exhibit a ring-like eyewall with both deep convection and shallow precipitation at the onset of RI (Kieper and Jiang 2012; Rogers et al. 2013b). The RI group of TCs also have a higher degree of axisymmetry in precipitation before and during the RI (e.g., Harnos and Nesbitt 2011, 2016a; Zagrodnik and Jiang 2014; Tao and Jiang 2015). Intense convection and total rainfall also increase after RI, which is likely a response to the RI process (Tao et al. 2017). Airborne Doppler radar observations indicate that intensifying TCs have a more complete eyewall structure with more convection inside the RMW than that of the nonintensifying storms (Rogers et al. 2013b, 2016; Zawislak et al. 2016). Using a symmetry index for rain and convection, Alvey et al. (2015) found that TCs with higher intensification rates (including those with RI) tend to have greater areal coverage of rainfall as well as a higher rate of symmetrization before and during their intensification (also Zawislak et al. 2016; Tao et al. 2017).

Kossin and Schubert (2001) and Hendricks et al. (2009) showed the mixing of potential vorticity (PV) into the eye from the eyewall PV ring by barotropic instability. The mixing of PV leads to a drop in the minimum sea level pressure similar to that in RI. This suggests that RI can be due to the internal dynamics under favorable environmental conditions. Within the framework of an axisymmetric and balanced vortex, Schubert and Hack (1982) suggested that both the vortex structure and the location of diabatic heating play crucial roles in the intensification of TCs. Vigh and Schubert (2009) found that the warm core in TCs may rapidly develop if a portion of the deep convection occurs in the inner-core region. Latent heat release that occurs inside the radius of maximum wind (RMW), or in regions of high inertial stability, can intensify the tangential wind more efficiently since low-level inflow and a spinup is generated at the RMW (Shapiro and Willoughby 1982; Pendergrass and Willoughby 2009; Rogers et al. 2013b, 2016; Smith and Montgomery 2016; Paull et al. 2017).

In summary, the RI process involves factors across a wide range of scales, including environmental conditions at the synoptic scale, the vortex structure at the mesoscale, and diabatic heating at the convective scale
(Marks and Shay 1998; Bister and Emanuel 1998; Wang and $\mathrm{Wu} 2004)$. In particular, factors such as the degree of symmetry, and the amount of deep convection inside of the RMW may be important for the internal dynamics of RI (Nolan et al. 2007; Rogers et al. 2013b, 2016; Zawislak et al. 2016). In the axisymmetric gradient wind balanced framework, Hack and Schubert (1986, hereafter HS86) studied the dynamic efficiency factor (DEF) with a prescribed uniform heating on an idealized Rankine vortex. The DEF measures the effectiveness of convective heating at any point in generating kinetic energy (KE). The DEF is large in the presence of eye warming and strong baroclinicity in the inner-core region (HS86).

Supertyphoon Haiyan (2013), the most intense tropical cyclone (TC) in the Western Hemisphere, experienced a period of RI where the maximum wind speed increased by $31 \mathrm{~m} \mathrm{~s}^{-1}$ in $24 \mathrm{~h}$. We use a $2-\mathrm{km}$ horizontal resolution full-physics cloud-resolving model to study the RI of Haiyan with the DEF analysis for internal dynamics. We emphasize the structural changes in the inner-core region observed during the RI period, including the axisymmetrization, contraction of the RMW, eyewall deep convection, increase in the DEF, boundary layer (BL) radial inflow, and the dramatic increase of $V_{\max }$. Our goal is to investigate the internal dynamics of RI with the DEF analysis. Section 2 describes the observations of Haiyan, the numerical model framework, and the DEF diagnostic theory. A comparison of simulations with the observations and an analysis of the axisymmetrization process of the vortex are presented in section 3. Section 4 describes the DEF diagnosis and the internal nonlinear dynamics of the inner-core region. Conclusions are given in section 5 .

\section{Observations, model description, and the dynamic efficiency factor}

\section{a. Observations}

Supertyphoon Haiyan formed on 2 November 2013 in the WNP from a westward-moving tropical disturbance embedded in a mixed Rossby-gravity wave (Shu and Zhang 2015). Haiyan developed in an environment with ample upper-ocean heat content, weak vertical wind shear, and favorable upper-level divergence (Lin et al. 2014). During the course of its westward movement before landfall (Fig. 1a), it underwent RI on 4-5 November to reach supertyphoon status $\left(\geq 67 \mathrm{~m} \mathrm{~s}^{-1}\right)$ based on the Joint Typhoon Warning Center (JTWC) best track data (Fig. 1b), and the intensity increased by as much as $31 \mathrm{~m} \mathrm{~s}^{-1}$ in $24 \mathrm{~h}$ starting 0000 UTC 5 November. Such an intensification rate is more than 1.5 times the RI criterion $\left(19.5 \mathrm{~m} \mathrm{~s}^{-1}\right.$ in $\left.24 \mathrm{~h}\right)$ in the WNP. 
(a) Haiyan_track \& Sea surface temperature
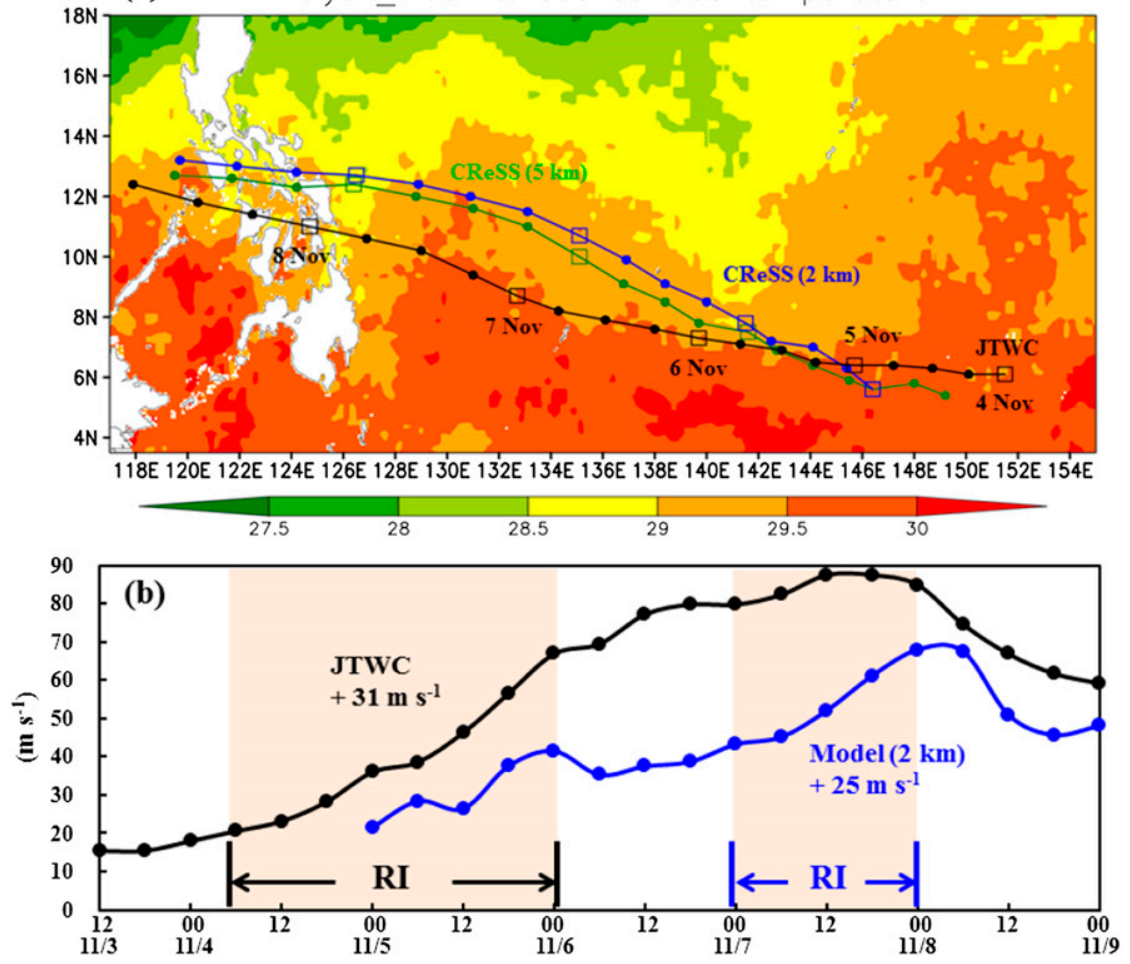

FIG. 1. (a) The SST $\left({ }^{\circ} \mathrm{C}\right.$, color) and the track of Typhoon Haiyan (2013): The JTWC best track (black) and the simulated tracks from the 5-km (green) and 2-km (blue) CReSS. The typhoon positions are given every $6 \mathrm{~h}$ (dots) and those at 0000 UTC are marked by open squares. (b) Time series of Haiyan intensity, in peak surface wind $\left(\mathrm{m} \mathrm{s}^{-1}\right)$, in JTWC and the 2-km CReSS simulations (blue at 10-m height). The shaded areas mark the observed and simulated periods of RI, and the maximum 24-h intensity changes are also labeled $\left(+25\right.$ to $+31 \mathrm{~m} \mathrm{~s}^{-1}$, on 5 Nov for JTWC).

Roughly $10 \mathrm{~h}$ before the initiation of RI, the Special Sensor Microwave Imager/Sounder (SSMIS) brightness temperature $\left(T_{B}\right)$ observations from polar-orbit satellites (Sun and Weng 2008) at 1941 UTC 3 November showed that the deep convection was only loosely organized and mostly located to the south of the storm center (Fig. 2a). At 0954 UTC 4 November, the eyewall convection became more concentrated at this early stage of RI (Fig. 2b). Later in the day at 2235 UTC when the RI was well underway, size contraction and a complete eyewall had organized with spiral bands (Fig. 2c). Finally, toward the end of the RI at 2056 UTC 5 November (Fig. 2d), the eye of Haiyan became even more axisymmetric with more deep convection compared to that at earlier times. We will show in the next section that the simulated RI resembles the structural characteristics shown in these observations.

\section{b. The CReSS model and experiments}

In this study, the Cloud-Resolving Storm Simulator (CReSS, version 3.4.3) developed at Nagoya University,
Japan (Tsuboki and Sakakibara 2002, 2007; Tsuboki 2008 ) is used to simulate Haiyan with its output used for the DEF diagnosis. CReSS is a nonhydrostatic and compressible cloud-resolving model that uses Cartesian and terrain-following height coordinates with a single domain. The cloud microphysics scheme has six species: vapor, cloud water, cloud ice, rain, snow, and graupel with double moment for the ice phase and single moment for the liquid phase. Parameterization for subgrid-scale turbulent mixing processes in the planetary boundary layer and surface radiation and heat/momentum fluxes are used as in Tsuboki and Sakakibara (2007). The CReSS model has been used to study TCs (e.g., Akter and Tsuboki 2012; Nomura and Tsuboki 2012; Wang et al. 2012, 2013, 2015a,b; Tsuboki et al. 2015; Kanada et al. 2017; Tsujino et al. 2017) as well as a variety of convective systems (e.g., Liu et al. 2004; Shimizu et al. 2008; Rafiuddin et al. 2013; Wang et al. 2014, 2016).

Two experiments have been performed using longitudelatitude coordinates and an offline-nesting method 

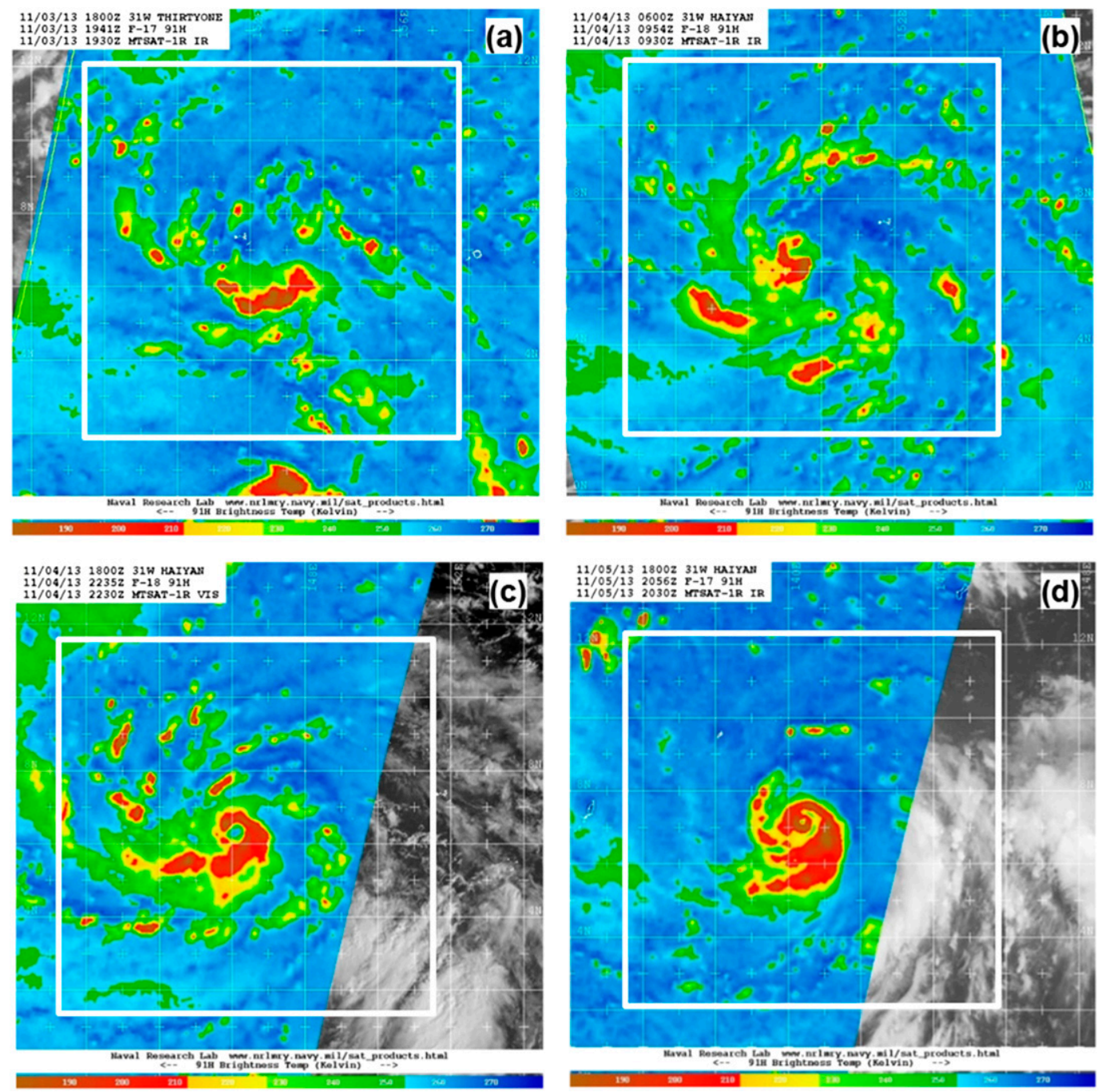

FIG. 2. SSMIS cloud imagery $\left(T_{B}, \mathrm{~K}\right)$ of Typhoon Haiyan (2013) at (a) 1941 UTC 3 Nov, (b) 0954 and (c) 2235 UTC 4 Nov, and (d) 2056 UTC 5 Nov 2013, overlaid on selected MTSAT VIS or IR imagery at the closest time (as labeled in upper-left corner). The images are from the Naval Research Laboratory. The white boxes correspond to the square areas shown in Fig. 4.

similar to Wang et al. (2011). The first is a 5-km run (with 80 vertical levels) that uses the Japan Meteorological Agency (JMA) $0.5^{\circ} \times 0.5^{\circ}$ global analyses (Kadowaki and Yoshimoto 2012) as the initial conditions and boundary conditions to simulate Haiyan for 5 days from 1200 UTC 4 November to 1200 UTC 9 November 2013, in a domain of roughly $57^{\circ}$ longitude $\times 24^{\circ}$ latitude in size. The JMA Merged Global Daily SST (MGDSST) analyses, which assimilate both satellite and in situ observations at a resolution of $0.25^{\circ} \times 0.25^{\circ}$, are used at the lower boundary. The output from the $5-\mathrm{km}$ run is then used to drive the second, 2-km experiment (with 76 vertical levels), which is designed to provide highresolution results for the DEF diagnosis. The 2-km simulation covers a slightly smaller area (about $48^{\circ}$ longitude $\times 21^{\circ}$ latitude) over a 4-day period starting from 0000 UTC 5 November 2013, with a model output interval of $5 \mathrm{~min}$.

\section{c. Equation for the dynamic efficiency factor}

We begin the derivation of the DEF with the axisymmetric governing equations for radial motion, tangential motion, hydrostatic balance, continuity, and thermodynamics:

$$
\begin{aligned}
\left(f+\frac{v}{r}\right) v & =\frac{\partial \phi}{\partial r}, \\
\frac{d v}{d t}+f u+\frac{u v}{r} & =F
\end{aligned}
$$




$$
\begin{aligned}
\frac{\partial \phi}{\partial z} & =\frac{g}{\theta_{0}} \theta, \\
\frac{\partial r u}{r \partial r}+\frac{\partial \rho w}{\rho \partial z} & =0, \\
\frac{d \theta}{d t} & =\frac{\partial \theta}{\partial t}+u \frac{\partial \theta}{\partial r}+w \frac{\partial \theta}{\partial z}=Q,
\end{aligned}
$$

where $u$ and $v$ are the radial and tangential wind components, respectively; $z=\left(c_{p} \theta_{0} / g\right)\left[1-\left(p / p_{0}\right)^{\kappa}\right]$ is the pseudoheight vertical coordinate; $w$ is the vertical velocity; $\rho=\rho_{0}\left(p / p_{0}\right)^{(1 / \kappa)-1}$ is the pseudodensity; $F$ is the forcing in tangential direction; $\phi$ is the geopotential; and $Q$ is the diabatic heating rate, with all other variables having their usual meaning.

Since $u=d r / d t$, the lhs of (1b) can be multiplied by $r$ to obtain

$$
r \frac{d v}{d t}+f r \frac{d r}{d t}+v \frac{d r}{d t}=\frac{d}{d t}\left(r v+\frac{1}{2} f r^{2}\right)=\frac{d m}{d t},
$$

where $m=r v+1 / 2 f r^{2}$ is the absolute angular momentum. Using (2), the left-hand side of (1a) becomes

$$
\begin{aligned}
f v+\frac{v^{2}}{r} & =\frac{1}{r^{2}} v\left(r v+f r^{2}\right) \\
& =\frac{1}{r^{3}}\left(r v+\frac{1}{2} f r^{2}-\frac{1}{2} f r^{2}\right)\left(r v+\frac{1}{2} f r^{2}+\frac{1}{2} f r^{2}\right) \\
& =\frac{1}{r^{3}}\left(m^{2}-\frac{1}{4} f^{2} r^{4}\right) .
\end{aligned}
$$

Thus, (1a) and (1b) can be rewritten as

$$
\begin{aligned}
& \frac{\partial \phi}{\partial r}=\frac{1}{r^{3}}\left(m^{2}-\frac{1}{4} f^{2} r^{4}\right) \\
& \frac{d m}{d t}=\frac{\partial m}{\partial t}+u \frac{\partial m}{\partial r}+w \frac{\partial m}{\partial z}=r F .
\end{aligned}
$$

By combining (4a) and (1c), the thermal-wind equation and its time derivative can be written as

$$
\begin{gathered}
\frac{g}{\theta_{0}} \frac{\partial \theta}{\partial r}=\frac{1}{r^{3}} \frac{\partial m^{2}}{\partial z}, \\
\frac{\partial}{\partial t}\left(\frac{g}{\theta_{0}} \frac{\partial \theta}{\partial r}\right)=\frac{\partial}{\partial t}\left(\frac{1}{r^{3}} \frac{\partial m^{2}}{\partial z}\right) .
\end{gathered}
$$

The static stability, baroclinicity, and inertial stability can be, respectively, defined as

$$
\rho A:=\frac{g}{\theta_{0}} \frac{\partial \theta}{\partial z},
$$

$$
\begin{aligned}
& \rho B:=-\frac{g}{\theta_{0}} \frac{\partial \theta}{\partial r}=-\frac{1}{r^{3}} \frac{\partial m^{2}}{\partial z}, \\
& \rho C:=\left(f+\frac{2 v}{r}\right)\left(f+\frac{\partial r v}{r \partial r}\right)=\frac{1}{r^{3}} \frac{\partial m^{2}}{\partial r} .
\end{aligned}
$$

Using these definitions, (4b) and (1e) can be expressed as

$$
\begin{gathered}
\frac{1}{r^{3}} \frac{\partial m^{2}}{\partial t}+\rho u C-\rho w B=\frac{2 m F}{r^{2}}, \\
\frac{g}{\theta_{0}} \frac{\partial \theta}{\partial t}-\rho u B+\rho w A=\frac{g}{\theta_{0}} Q .
\end{gathered}
$$

Next, based on the continuity in (1d), the 2D streamfunction $\psi$ (on the radius-height plane) is defined as

$$
(\rho u, \rho w)=\left[-\frac{\partial \psi}{\partial z}, \frac{\partial(r \psi)}{r \partial r}\right] .
$$

By taking the partial derivatives of (8a) with respect to $z$ and (8b) with respect to $r$ then subtracting them to eliminate the time-tendency terms using (6), the Sawyer-Eliassen circulation diagnostic equation (Eliassen 1952; Vigh and Schubert 2009; Bui et al. 2009) can be obtained as

$$
\begin{aligned}
\boldsymbol{L} \psi & =\frac{g}{\theta_{0}} \frac{\partial Q}{\partial r}-\frac{1}{r^{2}} \frac{\partial(2 m F)}{\partial z}, \text { where } \\
\boldsymbol{L}(\cdot) & =\frac{\partial}{\partial r}\left[A \frac{\partial r(\cdot)}{r \partial r}+B \frac{\partial(\cdot)}{\partial z}\right]+\frac{\partial}{\partial z}\left[B \frac{\partial r(\cdot)}{r \partial r}+C \frac{\partial(\cdot)}{\partial z}\right] .
\end{aligned}
$$

The operator (10b) is elliptic if $A C-B^{2}>0$. The boundary conditions of (10) are that $\psi=0$ on the top, bottom, and inner boundaries (at $r=0$ ), and that $\psi \rightarrow 0$ as $r \rightarrow \infty$.

The following two energy equations can be derived from (1) as

$$
\begin{aligned}
& \frac{d P}{d t}=H-C, \\
& \frac{d K}{d t}=C+M,
\end{aligned}
$$

where $P$ and $K$ denote the total potential and kinetic energies of the atmosphere, respectively; $H$ is the total heating; $C$ is the rate of conversion of $P$ into $K$; and $M$ is the KE-generation rate by momentum forcing; and

$$
\begin{aligned}
P & :=\iint c_{p} T \rho r d r d z, \\
K & :=\iint \frac{v^{2}}{2} \rho r d r d z,
\end{aligned}
$$




$$
\begin{aligned}
H & :=\iint c_{p} \Pi Q \rho r d r d z, \\
C & :=\iint \frac{g}{\theta_{0}} w \theta \rho r d r d z, \quad \text { and } \\
M & :=\iint F v \rho r d r d z,
\end{aligned}
$$

where $c_{p}$ is specific heat at constant pressure and $\Pi=$ $T / \theta=\left(p / p_{0}\right)^{\kappa}$ is the nondimensional pressure. Now, we use (9) to rewrite (12d) as

$$
C=\iint \frac{g}{\theta_{0}} \theta \frac{\partial r \psi}{\partial r} d r d z
$$

Through integration by parts with the boundary conditions, (13) becomes

$$
C=-\iint \psi \frac{g}{\theta_{0}} \frac{\partial \theta}{\partial r} r d r d z
$$

Now, define a variable $\chi$ to be the solution of

$$
\boldsymbol{L}_{\chi}=\frac{g}{\theta_{0}} \frac{\partial \theta}{\partial r}
$$

with similar boundary conditions for $\chi$ as those of (10). Substituting (15) into (14) and applying the self-adjoint property of the linear operator $\boldsymbol{L}$ (which remains elliptic for a stable vortex) with the $\psi=0$ boundary conditions, we obtain

$$
C=-\iint \psi \boldsymbol{L} \chi r d r d z=-\iint \chi \boldsymbol{L} \psi r d r d z .
$$

Using (10a) in (16) and performing a final integration by parts, we obtain

$$
\begin{aligned}
C & =-\iint \chi\left[\frac{g}{\theta_{0}} \frac{\partial Q}{\partial r}-\frac{1}{r^{2}} \frac{\partial(2 m F)}{\partial z}\right] r d r d z \\
& =\iint \frac{g}{\theta_{0}} Q \frac{\partial r \chi}{r \partial r} r d r d z-\iint \frac{2 m}{r^{2}} F \frac{\partial \chi}{\partial z} r d r d z \\
& =\iint \eta_{H} c_{p} \Pi Q \rho r d r d z+\iint \eta_{M} F v \rho r d r d z,
\end{aligned}
$$

where

$$
\begin{aligned}
& \eta_{H}=\frac{g}{\rho c_{p} \Pi \theta_{0}} \frac{\partial r \chi}{r \partial r} \\
& \eta_{M}=-\frac{2 m}{\rho v r^{2}} \frac{\partial \chi}{\partial z} .
\end{aligned}
$$

Here, we define $\eta_{H}$ as the DEF of convective heating and $\eta_{M}$ as the DEF of momentum, and they represent the efficiency of converting diabatic forcing and momentum forcing to KE of the balanced flow. Our simulations indicate that the momentum term is one order of magnitude smaller than the heating term in (17) in the inner core, thus only the DEF of convective heating $\eta_{H}$ is considered further in present manuscript. For the entire TC, the system DEF $\left(\bar{\eta}_{H}\right)$, or the ratio of conversion term to the diabatic heating term, is defined as

$$
\bar{\eta}_{H}=\frac{C}{H}=\frac{\iint \eta_{H} Q c_{p} \Pi \rho r d r d z}{\iint Q c_{p} \Pi \rho r d r d z} .
$$

The system DEF measures the effectiveness of the convective heating in producing $\mathrm{KE}$ in the $\mathrm{TC}$ as a whole.

Since Haiyan exhibits little vertical tilt throughout the period of interest, a method based on Braun (2002) is used to identify its center (Tsujino et al. 2017). After converting model output from Cartesian to cylindrical coordinates following the TC center, static stability $(\rho A)$, baroclinicity $(\rho B)$, and inertial stability $(\rho C)$ are computed using (7). Then, (15) is used to solve $\chi$ $(0 \leq r \leq 1000 \mathrm{~km}$ and $1.5 \leq z \leq 13.2 \mathrm{~km})$. The dynamic efficiency factor of heat $\left(\eta_{H}\right)$ computed using (18a), and the efficiency of the entire system $\left(\bar{\eta}_{H}\right)$ using (19) with the CReSS model output $Q$.

\section{Model simulation results}

The simulated tracks of the 2-km experiments deviate to the north of the JTWC best track beginning at 0000 UTC 6 November (Fig. 1a). This may be due to a weaker subtropical high with a position too far north in the model simulation. The averaged track error is $241 \mathrm{~km}$, which results in a slightly cooler SST environment $\left(\sim 0.5^{\circ} \mathrm{C}\right.$.) Since Haiyan remained over warm water of at least $29^{\circ} \mathrm{C}$ and was fast moving $\left(\sim 9 \mathrm{~m} \mathrm{~s}^{-1}\right)$ during its RI stage, the negative ocean feedback effect was minimal (Lin et al. 2014) and thus such a small difference in SST is expected to have only a limited effect on the energy supply from the ocean.

Our 2-km simulation reproduces the RI of Haiyan, although the timing is delayed by about $48 \mathrm{~h}$ and the peak intensity is weaker than that observed (Fig. 1b). In the JTWC best track data, Haiyan intensified by $31 \mathrm{~m} \mathrm{~s}^{-1}$ (from 36 to $67 \mathrm{~m} \mathrm{~s}^{-1}$ ) during 0000-2400 UTC 5 November, while the $2-\mathrm{km}$ experiment had a $25 \mathrm{~m} \mathrm{~s}^{-1}$ $\left(43-68 \mathrm{~m} \mathrm{~s}^{-1}\right)$ increase in $24 \mathrm{~h}$ starting at 0000 UTC 7 November. Thus, both the initial/final intensities and the rate of RI (for the 24-h period of our main interest) are in general agreement. The delayed occurrence of the 
$\mathrm{RI}$ in CReSS is likely related to the fact that the model needs an adjustment period to generate the correct vortex strength in the initial field. The peak intensity of $71-74 \mathrm{~m} \mathrm{~s}^{-1}$ near landfall is also less than the estimate by JTWC (Fig. 1b).

The time series of the vertical wind shear between 850 and $200 \mathrm{hPa}$ (within $r=1000 \mathrm{~km}$ ) associated with the TC in the 2-km simulation is shown in Fig. 3, which can be compared with the observation in Fig. S8 of Lin et al. (2014). While the observed RI occurred during 4-5 November when Haiyan experienced an increase in the vertical shear from about 6 to $10 \mathrm{~m} \mathrm{~s}^{-1}$ (Lin et al. 2014), the TC in the model encounters a shear of less than $5-6 \mathrm{~m} \mathrm{~s}^{-1}$ throughout its RI period (on 7 November, Fig. 3) and thus a condition even more favorable for intensification. Hendricks et al. (2010) found the mean deep-layer vertical shear for TCs that experience RI in the western Pacific to be approximately $10 \mathrm{~m} \mathrm{~s}^{-1}$, suggesting that the shear conditions are still quite favorable even for the observed RI event in Haiyan.

Figure 4 shows the mixing ratio of precipitation (rain, snow, plus graupel), vertically averaged over $850-200 \mathrm{hPa}$, surrounding the TC center at 1200 UTC 6 November before RI, at 0000 and 0600 UTC 7 November during the early stage of RI, and at 0000 UTC 8 November at the late stage of RI in the simulation. The deep convection associated with RI around the inner core of the TC compares favorably with the SSMIS observations in Fig. 2. In particular, the asymmetry before the RI (Fig. 4a), the establishment of an eyewall, its contraction (Figs. 4b and 4c) during the RI, and the symmetric structure toward the later stage of RI (Fig. 4d) are simulated. Even though the inner eye is not as small and tight compared to Fig. 2d, the 2-km simulation does produce an RI process that resembles the observations, and therefore its results are further used to calculate the DEF diagnostics.

Consistent with many earlier studies on the distribution of convection in the RI phase (Kieper and Jiang 2012; Rogers et al. 2013b; Zagrodnik and Jiang 2014; Alvey et al. 2015; Tao and Jiang 2015), both satellite observation and model simulation show that the RI of Haiyan is accompanied by the establishment of the eyewall and a more axisymmetric storm (Figs. 2 and 4). To better understand how this axisymmetrization relates to the dynamic efficiency of latent heat release, we analyze the evolution in the degree of axisymmetric structure of Haiyan in the 2-km experiment quantitatively, by using the axisymmetry parameter $\gamma$ of Miyamoto and Takemi (2013). Any model variable $\phi$ can be separated into an azimuthal-mean $\bar{\phi}$ and the deviation from this mean $\phi^{\prime}$, as

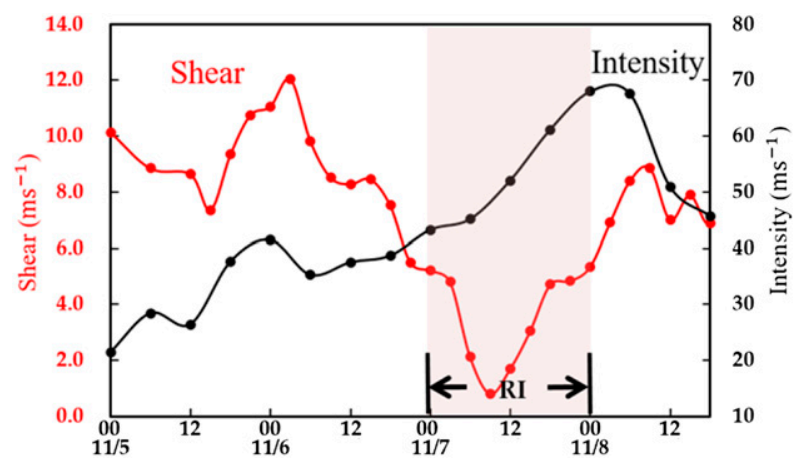

FIG. 3. Time series of typhoon intensity $\left(\mathrm{m} \mathrm{s}^{-1}\right.$, black, as in Fig. 1b) and environmental vertical wind shear (in $\mathrm{m} \mathrm{s}^{-1}$, red) simulated by the $2-\mathrm{km}$ CReSS model. The shaded area marks the RI period of interest in the model.

$$
\phi(r, \lambda, z, t)=\bar{\phi}(r, z, t)+\phi^{\prime}(r, \lambda, z, t)
$$

where $\lambda$ is the azimuthal direction and all other variables have the same meaning as before. The axisymmetry parameter $\gamma$ is defined as (Miyamoto and Takemi 2013)

$$
\gamma(r, z, t) \equiv \frac{\bar{\phi}(r, z, t)^{2}}{\bar{\phi}(r, z, t)^{2}+\int_{0}^{2 \pi} \phi^{\prime}(r, \lambda, z, t)^{2} d \lambda / 2 \pi}
$$

and it represents the reciprocal of the fraction of the deviation component at its corresponding radius, and reflects the degree of axisymmetric structure. The value of $\gamma$ equals 1 when $\phi$ is perfectly axisymmetric, and close to 0 when $\phi$ is highly asymmetric.

For a cylindrical volume with a fixed radius $R$ and height $Z$ (above $1.5 \mathrm{~km}$ ), the averaged axisymmetricity $\bar{\gamma}^{R Z}$ at any instant can be computed as

$$
\bar{\gamma}^{R Z}(t)=\frac{1}{R Z} \sum_{z=1.5}^{Z} \sum_{r=0}^{R} \gamma(r, z, t)
$$

and we set $Z$ to $12 \mathrm{~km}$ and $R$ to $100 \mathrm{~km}$ to focus on the inner core of the TC. The dynamic and thermodynamic parameters chosen for $\bar{\gamma}^{R Z}$ include tangential wind, $\mathrm{PV}$, mixing ratio of precipitating hydrometeors, hourly rainfall, and diabatic heating rate.

Among the five time series of the selected variables in Fig. 5, tangential wind has the overall highest axisymmetry, followed by the vertical-averaged PV, hourly rainfall, precipitating hydrometeors, and 6-h mean diabatic heating near and during RI (Fig. 5). Compared to $\bar{\gamma}_{v}{ }^{R Z}$ (mostly $\geq 0.8$ ) and ${\overline{\gamma_{\text {dia }}}}^{R Z}$ (below 0.2 ), the other three axisymmetricity parameters show a more significant increase before and during RI. Among them, $\overline{\gamma_{\mathrm{PV}}} R Z$ for PV (black) increases from about 0.3 at 1800 UTC 5 November to 0.7 at 1200 UTC 8 November. 
(a) 1200 UTC 06 Nov.

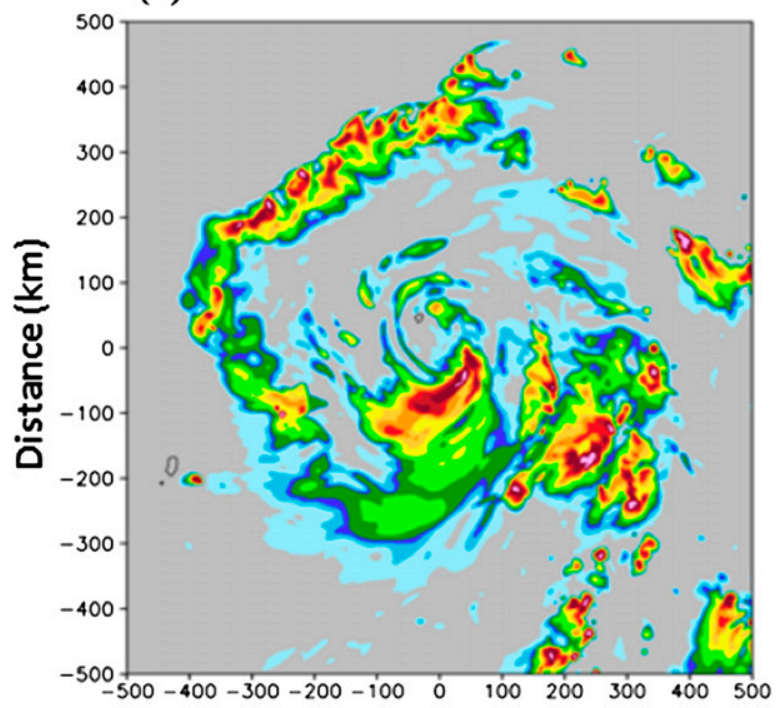

(c) 0600 UTC 07 Nov.

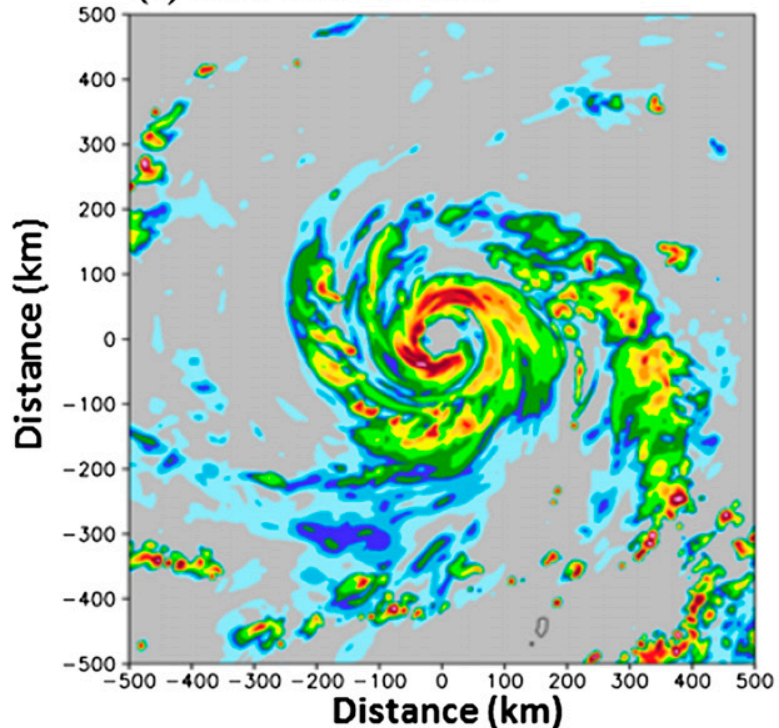

(b) 0000 UTC 07 Nov.

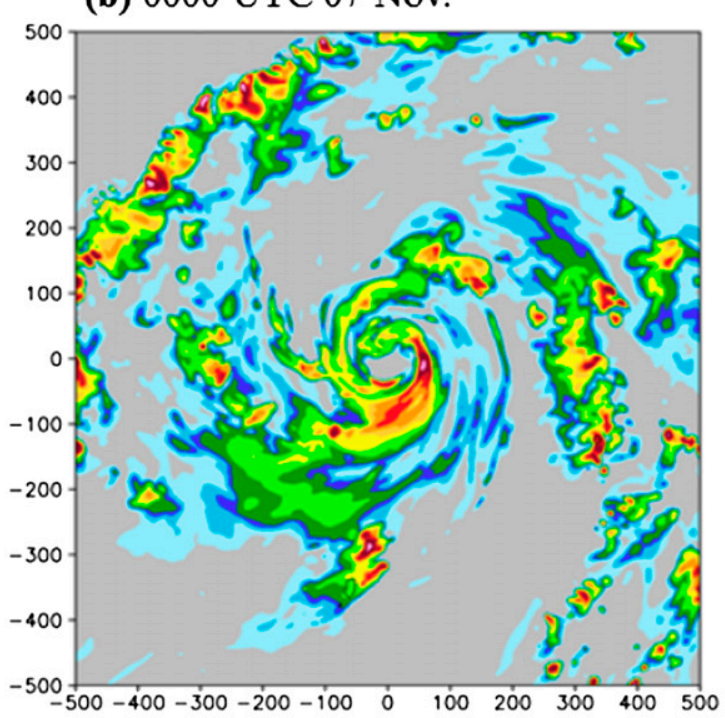

(d) 0000 UTC 08 Nov.

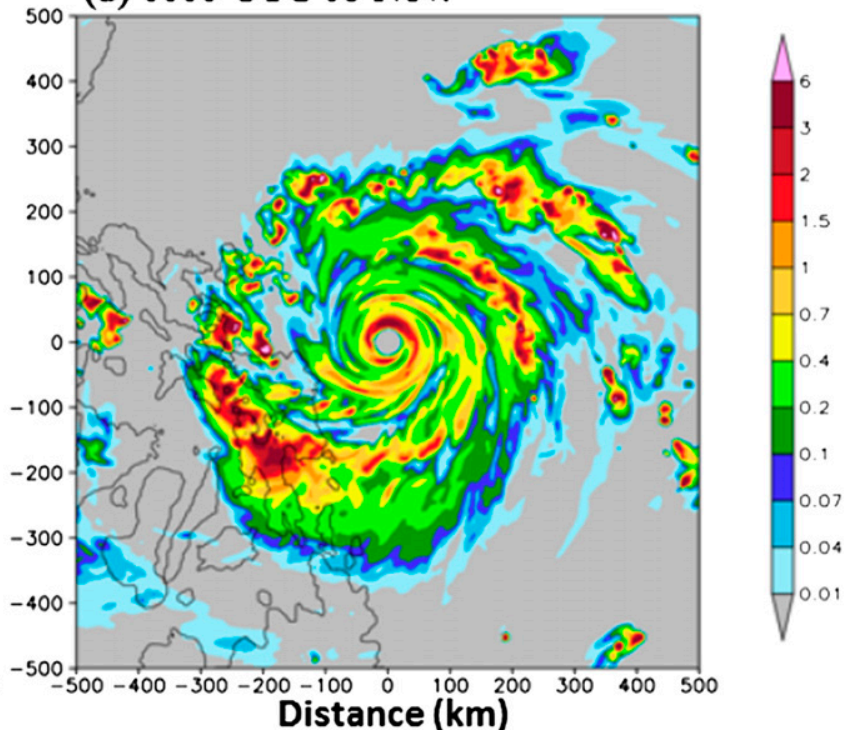

FIG. 4. Vertically-averaged mixing ratio of precipitating hydrometeors ( $\mathrm{g} \mathrm{kg}^{-1}$, color) over 850-200 hPa at (a) $1200 \mathrm{UTC} 6 \mathrm{Nov}$, (b) 0000 and (c) 0600 UTC 7 Nov, and (d) 0000 UTC 8 Nov 2013 in the $2-\mathrm{km}$ run, inside a square area of $1000 \times 1000 \mathrm{~km}^{2}$ centered on the TC.

An increase in axisymmetry in PV prior to RI was also found by Miyamoto and Takemi (2015) in their idealized TC simulations. Consistent with previous studies (Guimond et al. 2010; Harnos and Nesbitt 2011; Rogers et al. 2013b; Zagrodnik and Jiang 2014; Alvey et al. 2015; Tao and Jiang 2015), the axisymmetry of rainfall also increases both before and during RI, and exceeds 0.6 near 0600 UTC 8 November.

Figure 6 shows the PV at 2.4-km altitude with vertical motion on top of BL $(1 \mathrm{~km})$, at four different times before (Fig. 6a), at (Fig. 6b), and after the onset of RI (Figs. 6c,d). Before the RI, the PV field in the TC appears in the shape of the hollow tower within the radius of $50 \mathrm{~km}$, in that the PV in the center is about $6 \mathrm{PV}$ unit (PVU; $1 \mathrm{PVU}=10^{-6} \mathrm{~K} \mathrm{~kg}^{-1} \mathrm{~m}^{2} \mathrm{~s}^{-1}$ ) surrounded with higher PV ring of some 15 PVUs. There are local high PV regions (20 PVUs) in the square to pentagonal PV configuration in the PV ring region (Fig. 6a). The local peak PV regions in the polygonal configuration are similar to that in the mesovortices in Hurricane Isabel (2003) (Kossin and Schubert 2004). Schubert et al. (1999) and Kossin and Schubert (2001; 2004) suggest that the mesovortices play an essential role in the TC intensification through PV mixing by the barotropic 


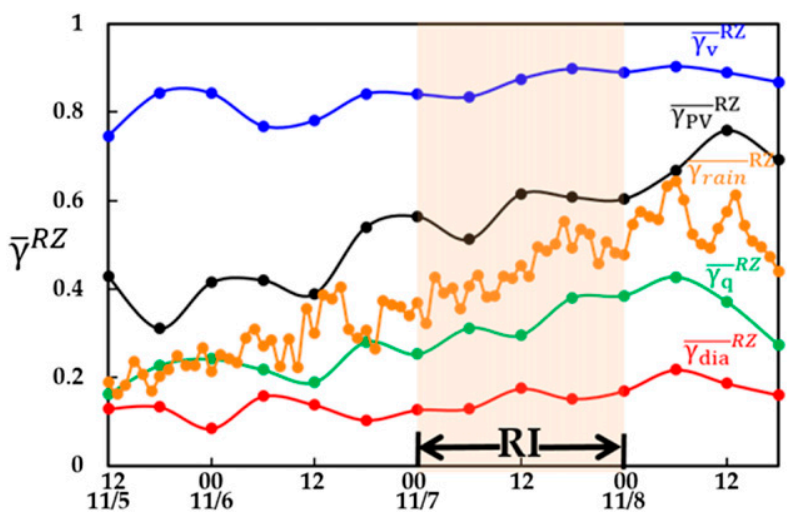

FIG. 5. Time series of the degree of axisymmetric structure $\left(\bar{\gamma}^{R Z}\right)$ of five selected parameters in the 2-km run: tangential wind speed $\left(\bar{\gamma}_{v}^{R Z}\right.$, blue $), 1$-h rainfall $\left(\bar{\gamma}_{\text {rain }} R Z\right.$, orange $), \mathrm{PV}\left({\overline{\gamma_{\mathrm{PV}}}}^{R Z}\right.$, black $)$, mixing ratio of precipitating hydrometeors $\left(\bar{\gamma}_{q} R Z\right.$, green $)$, and 6-h-averaged diabatic heating $\left(\bar{\gamma}_{\text {dia }} R Z\right.$, red, $\pm 3 \mathrm{~h}$ from the time indicated). The axisymmetry is dimensionless, and the shaded area depicts the RI period.

instability. The PV field mixing becomes more evident in Fig. $6 \mathrm{~b}$ with the structure appearing as a triangular shape, and a near monopole structure in the center at the later time (Fig. 6c). Eventually, the mixing leads to a monopole structure of $\mathrm{PV}$ of 50 PVUs in the core (Fig. 6d). The evolution of the polygonal structure of PV field at the $2.4 \mathrm{~km}$ resembles the vorticity mixing in the barotropic model of Schubert et al. (1999). The monopole PV in Fig. 6d, however, is stronger than that in the earlier time. This may be due to the presence of intense convective heating during the RI period. Note that the Fig. $6 \mathrm{~d}$ is the beginning of another phase of PV mixing as the large PV ring began to appear on the eyewall region of the TC. In fact, the monopole structure of $\mathrm{PV}$ is produced in the model at later time (not shown).

Note that the regions of BL top updraft in the eyewall also appeared in the polygonal configuration and are nearly locked with the mesovortices in Fig. 6a. Kuo et al. (2016) investigated the deep convection in the polygonal eyewall of TCs. They found from observations and slab BL model results that strong updrafts at BL top often occurred at the edge of the polygonal eyewall. The PV field at 2.4-km height and the BL top updraft at $1 \mathrm{~km}$ in Figs. $6 \mathrm{a}$ and $6 \mathrm{~b}$ are both in general agreement with the deep convection in the polygonal study of Kuo et al. (2016). During the RI period, the high PV area in the eyewall becomes more collocated with the eyewall convection (Fig. 6). This will lead to large PV production during the RI period. The increase of axisymmetrization prior to RI supports the usage of the axisymmetric DEF formula to understand the internal dynamics of RI.

\section{Diagnostics of dynamic efficiency of latent heat release}

\section{a. Analysis of TC structural factors}

As can be seen from (15) and (18), the DEF depends on the baroclinicity of the vortex and the instantaneous vortex structure through the coefficients $A, B$, and $C$. Figure 7 shows the distributions of the vortex static stability $\rho A$ within $100 \mathrm{~km}$ on the $r-z$ plane at the times indicated in Fig. 6, before (Fig. 7a), at (Fig. 7b), and after the onset of RI (Figs. 7c,d). The vortex static stability plotted here is with static stability of the environment removed. In the inner core $(r \leq 100 \mathrm{~km})$, there is a predominant stratified structure in the static stability with the most-stable layer near 5-6 km corresponding to the bottom of the warm core (Fig. 7a). The area of large static stability descends with time near the center. The decreasing altitude of the high static stability region corresponds to the warm core due to the increase of subsidence in the eye during RI. There is another region of warming that occurs at the radius of the model RMW (the RMW at the 1.5-km height) in the later stage of RI (Fig. 7d). The warming may be due to the strong subsidence associated with the eyewall deep convection. (The detailed structure of the subsidence is discussed along with baroclinicity in Fig. 9.)

The inertial stability $\rho C$ starts with low values and increases significantly near and inside the model RMW during RI (Fig. 8). The region of high inertial stability $\geq 3 \times 10^{-6} \mathrm{~s}^{-2}$ is confined inside $r=15 \mathrm{~km}$ (Fig. 8a). Figure $8 \mathrm{~b}$ has a somewhat similar $\rho C$ structure but with the contraction of the model RMW. The PV field in Fig. 8 shows a hollow PV structure before the onset of RI but becomes a monopole structure with high PV in the center at later times (Figs. $8 \mathrm{c}$ and $8 \mathrm{~d}$ ). This is consistent with the discussion of Fig. 6, which shows PV axisymmetrization and mixing in the lower troposphere. The above structural changes in the inertial stability and PV field are consistent with a contraction of the RMW before the onset of the RI. Finally, we note that the high inertial stability region of $20 \times 10^{-6} \mathrm{~s}^{-2}$, which coincides with the high PV region of 50 PVUs, expands upward and outward to $12 \mathrm{~km}$ (Fig. 8d) during the RI.

Figure 9 shows a dramatic increase of inner-core baroclinicity near the RMW during the RI. The outwardsloping updraft in the strong eyewall convection and the subsidence near the edge of the eyewall are most clearly seen in Fig. 9d. Figure 9d also indicates narrow and outward-leaning deep convection coupled with the outward-tilting region of high inertial stability and high PV (Fig. 8d). The deep convection, with an outwardsloping region of strong updraft, is locked with the large 
(a) PV (2.4 km) and W (1 km) 1800 UTC 06 Nov

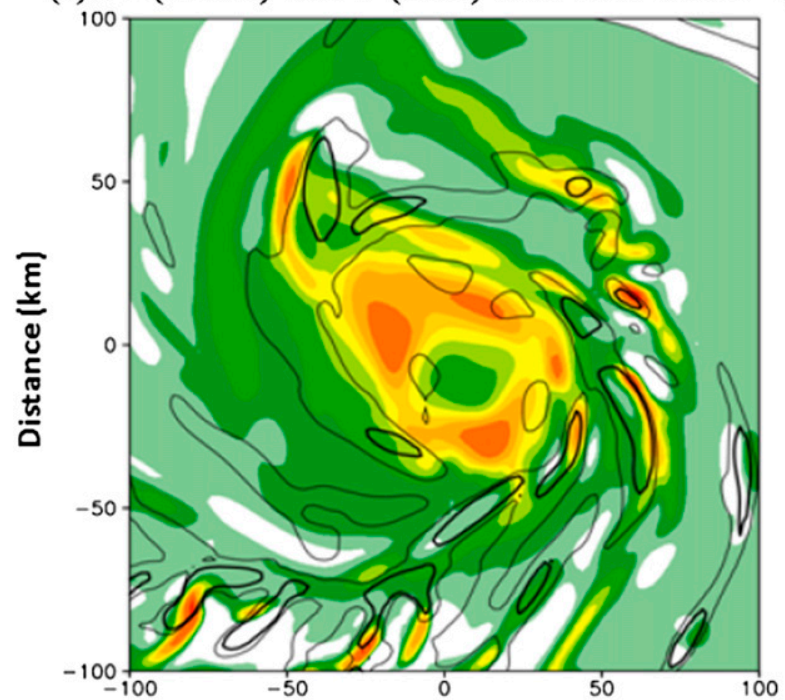

(c) PV (2.4 km) and W (1 km) 0600 UTC 07 Nov

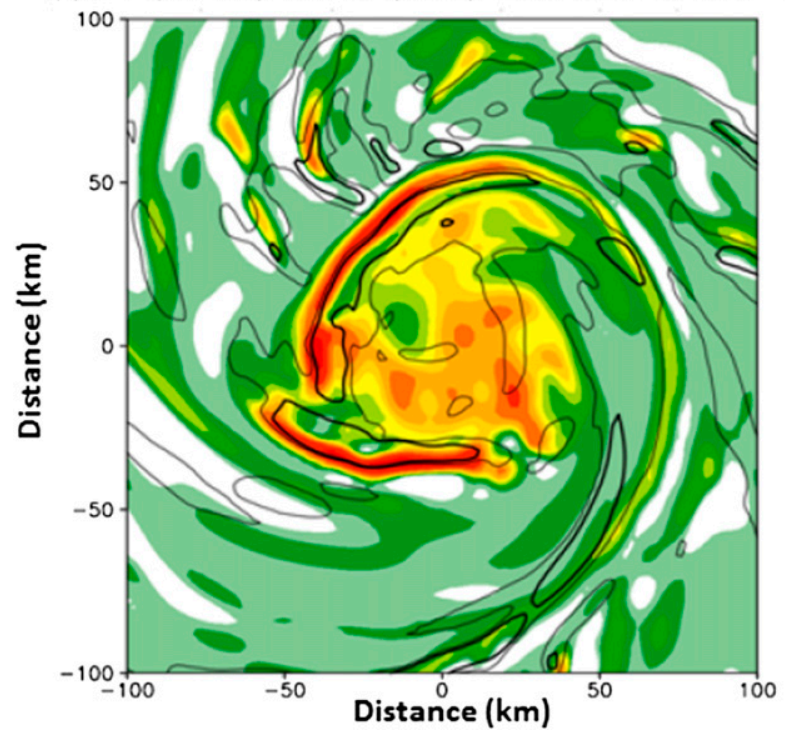

(b) PV $(2.4 \mathrm{~km})$ and W (1 km) 0000 UTC 07 Nov

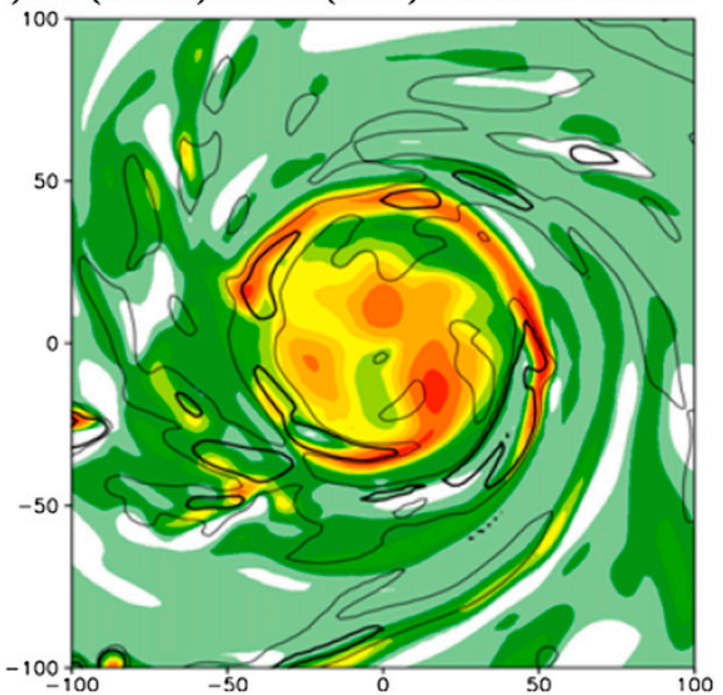

(d) PV $(2.4 \mathrm{~km})$ and W (1 km) 1800 UTC 07 Nov

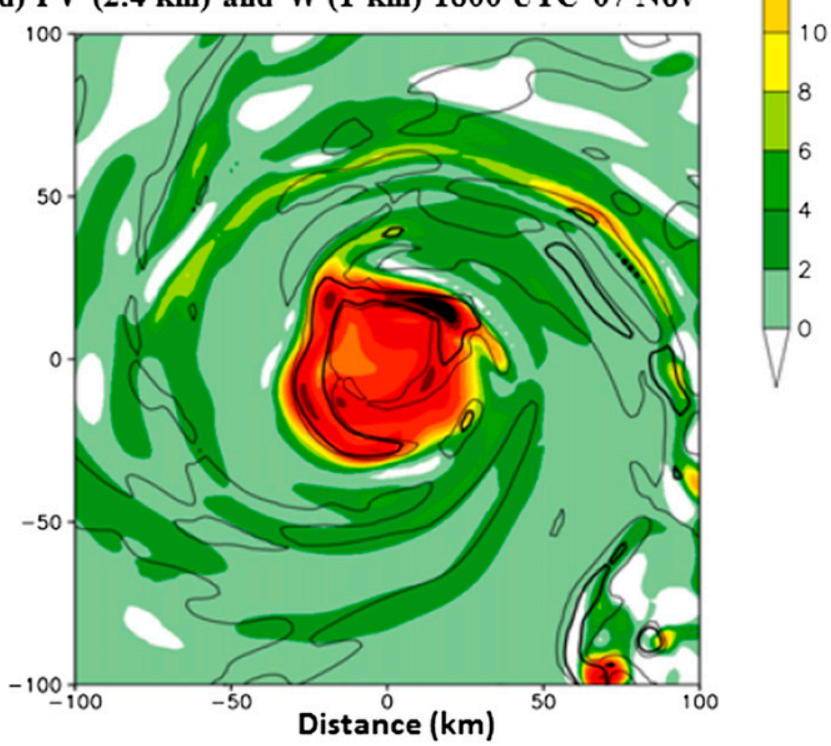

FIG. 6. Distribution of PV (PVU, 1 PVU $=10^{-6} \mathrm{~K} \mathrm{~m}^{2} \mathrm{~kg}^{-1} \mathrm{~s}^{-1}$; color) at a height of $2.4 \mathrm{~km}$ and vertical velocity $w$ (thin contour at $0.25 \mathrm{~m} \mathrm{~s}{ }^{-1}$ and thick contour at $1 \mathrm{~m} \mathrm{~s}^{-1}$ ) at a height of $1 \mathrm{~km}$ at (a) 1800 UTC 6 Nov, (b) 0000 UTC 7 Nov, (c) 0600 UTC 7 Nov, and (d) 1800 UTC 7 Nov 2013 in the 2-km simulation, inside a box of $200 \times 200 \mathrm{~km}^{2}$ centered on the TC. The onset time of RI is 0000 UTC 7 Nov 2013.

PV field. The result in Fig. 9 is similar to the high PV convective tower of Hausman et al. (2006) except that our model has a smaller PV value of about 50 PVUs. Hausman et al. (2006), with an axisymmetric cloudresolving model with a finest grid of $0.5-\mathrm{km}$ resolution, showed that the PV and convective heating become locked together in a narrow leaning convective tower on the inner edge of the eyewall. The thin, leaning convective tower is an efficient PV producing machine in which the PV value may reach well over 100 PVUs. The PV value is much larger than that in our model. The assumption of axisymmetry and the nearly inviscid flow without the PV mixing, however, is likely to produce overly intense PV values in their model. On the other hand, Bell et al. (2018) and Martinez et al. (2019, manuscript submitted to J. Atmos. Sci.) recently have done a PV analysis of Hurricane Patricia (2015), using high density dropsondes and radar. They find very high values of PV in the convective towers with peak values exceeding 250 PVU. The magnitude of PV in the convective tower may be model resolution dependent. Overall, the convective tower in Fig. 9d can be important for the PV production in the RI period. 
(a) $11 / 061800$ UTC
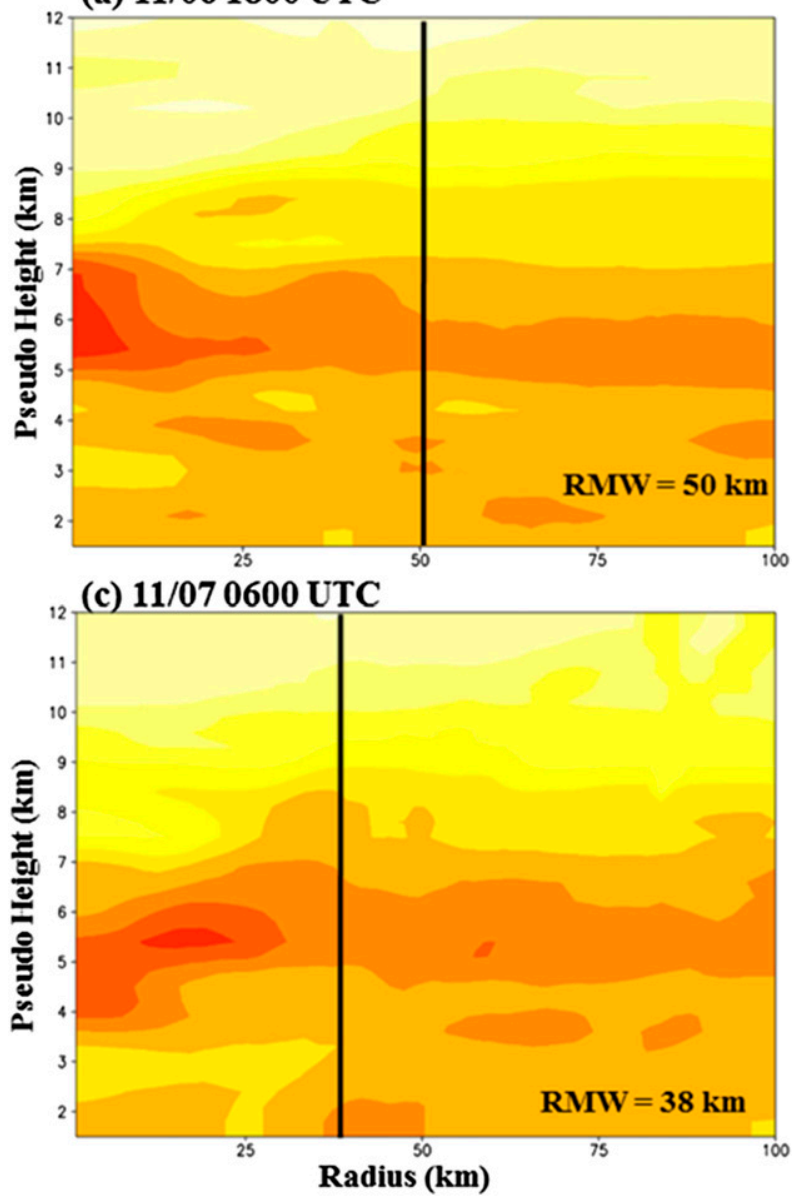

(b) 11/07 0000 UTC (RI onset)

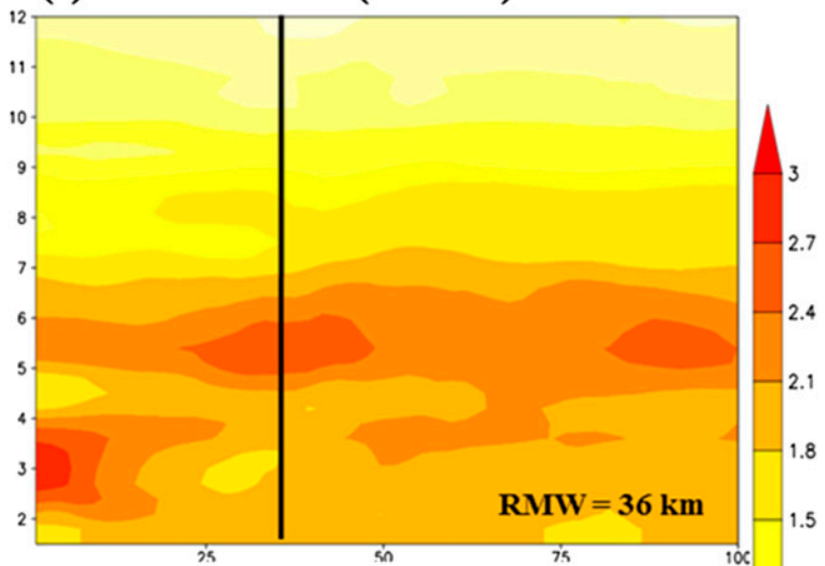

(d) $11 / 071800$ UTC

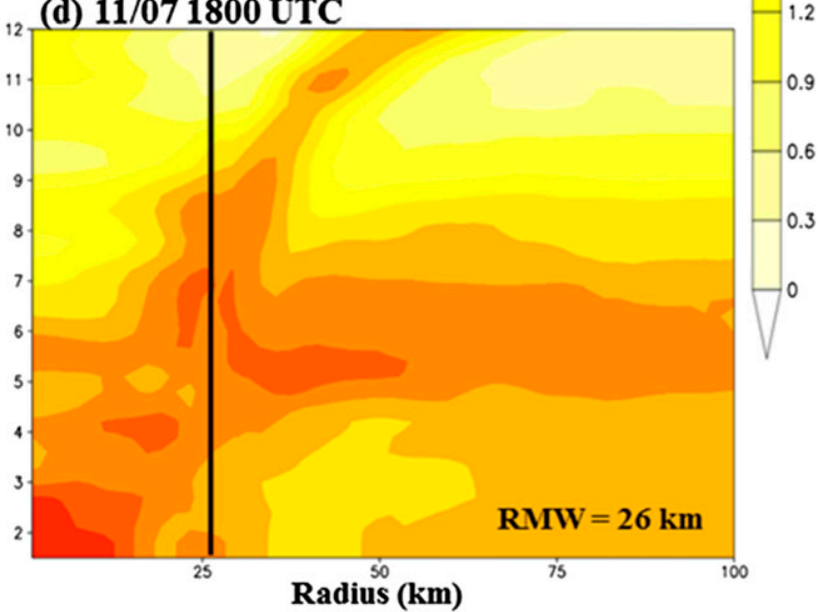

FIG. 7. Radius-height distribution of azimuthally averaged static stability $\rho A\left(10^{-4} \mathrm{~s}^{-2}\right)$ at (a) 1800 UTC 6 Nov, and (b) 0000 UTC, (c) 0600 UTC, and (d) 1800 UTC 7 Nov 2013 in the $2-\mathrm{km}$ simulation, for $r=0-100 \mathrm{~km}$ (TC center at $r=0$ ) and $z=1.5-12 \mathrm{~km}$. The vertical lines indicate the azimuthally averaged RMW at $1.5 \mathrm{~km}$ (also labeled); and the RI onset is at 0000 UTC 7 Nov 2013.

Figure 9 also shows that the subsidence is not uniform within the eye. Schubert et al. (2007) studied the distribution of the subsidence in the TC eye. When a storm is intense, the subsidence is larger at the inside edge of eyewall than at the center of the eye. The large subsidence at the inside edge of eyewall leads to a baroclinic reduction region in the center of the eye at $8-12 \mathrm{~km}$ (Figs. 9c and 9d). The baroclinicity increase near the eyewall, however, is larger in magnitude than that in the baroclinic reduction region. As we will show in Fig. 10, the overall eye warming by subsidence enhances the DEF in the eye and the eyewall. The increase of the DEF value in the eye region converts more KE from the convective heating during the RI period.

\section{b. Dynamic efficiency of latent heat release}

Figure 10 suggests that the average tangential wind (about $36 \mathrm{~m} \mathrm{~s}^{-1}$ at $1.5 \mathrm{~km}$ ) increases only gradually before the onset of RI at 0000 UTC 7 November (Figs. 10a,c) but much more rapidly afterward during the RI stage. The rapid increase of the tangential wind is in general agreement with the increase in inertial stability within the vortex core region (cf. Fig. 8). In addition, the vertical profile of the RMW becomes less tilted and more upright with time. Figure 10 also shows that the rapid increase of average diabatic heating $Q$ during RI and the deep convection is concentrated inside the model RMW. Near and inside the model RMW, the DEF generally increases toward the upper levels and can reach over $10 \%$. However, there may not be significant eyewall convection prior to the RI. During the RI period, deep convection inside the RMW extends upward and becomes better coupled with the dynamic efficiency $\eta_{H}$. The results shown in Fig. 10 highlight the importance of deep convection inside the RMW and the increase of DEF to the RI process. This is also in agreement with many earlier studies that deep convection inside the RMW support intensification (e.g., Montgomery et al. 2006; 
(a) 11/06 1800 UTC

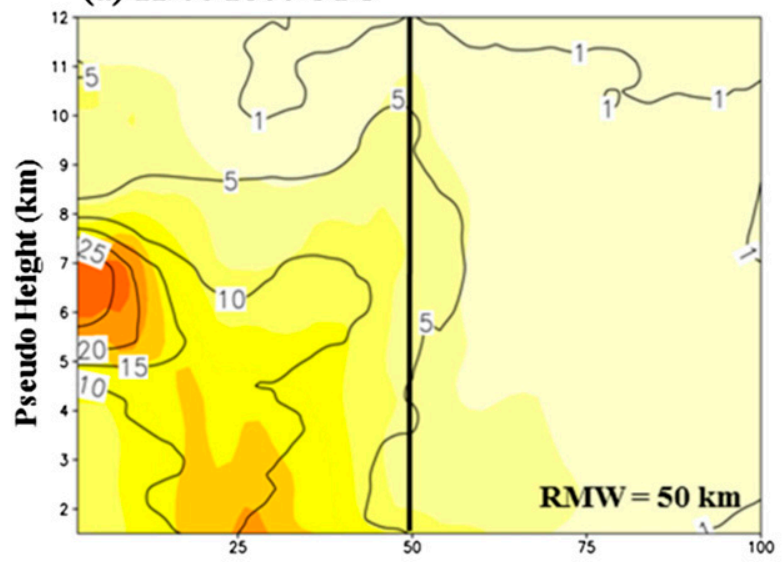

(c) $11 / 070600$ UTC

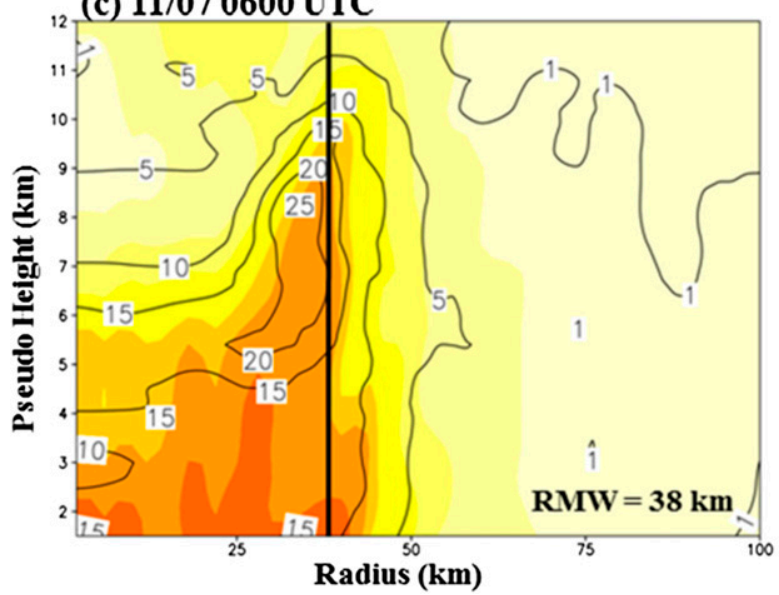

(b) 11/07 0000 UTC (RI onset)

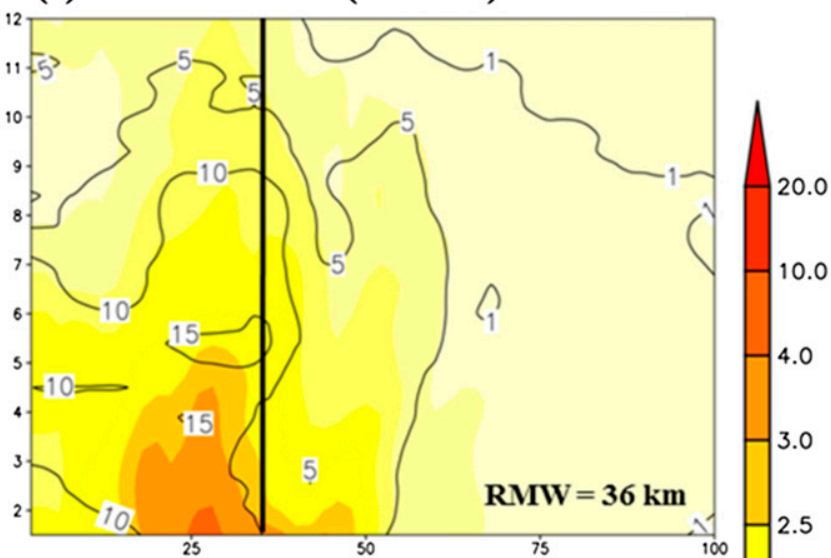

(d) $11 / 071800$ UTC

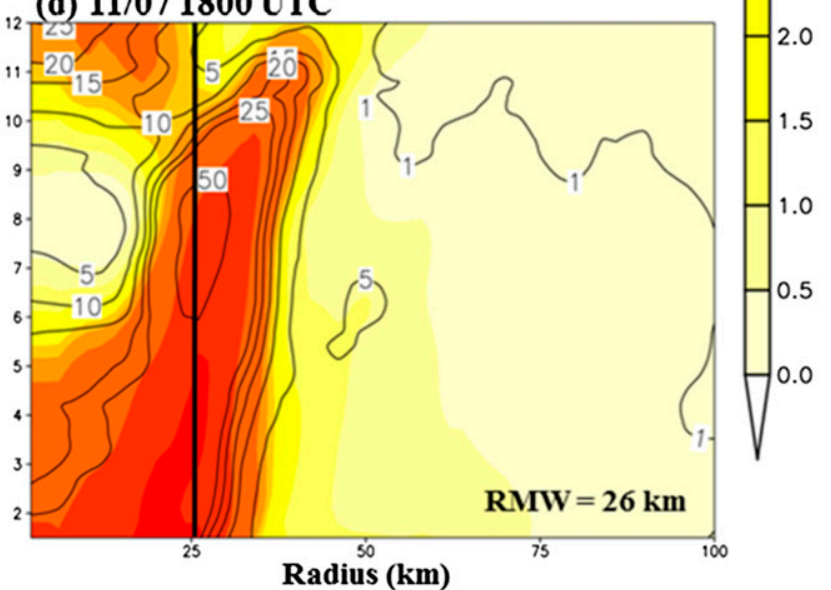

FIG. 8. As in Fig. 7, but showing the azimuthally averaged inertial stability $\rho C\left(10^{-6} \mathrm{~s}^{-2}\right.$, color) and PV (PVU, contour) in the 2-km simulation.

Kieper and Jiang 2012; Guimond et al. 2010, 2016; Rogers et al. 2013b, 2016; Zawislak et al. 2016; Harnos and Nesbitt 2016a,b).

Figure 11 illustrates the increase of inward radial flow in the BL during RI. This large unbalanced BL radial inflow exerts a strong control on the eyewall updraft and organization of deep convection. The inflow coupled with the absolute vorticity also leads to an increase in the tangential wind, and maximum tangential wind speed is found to occur within the inner-core BL (e.g., Fig. 11c). Note that the convective heating which results from the convergence of the radial inflow, occurs at a radius inside the RMW. A comprehensive review of the frictional $\mathrm{BL}$ control in the TC vortex evolution can be found in Montgomery and Smith (2017).

Since the high DEF values in the TC vortex is near the inner-core region and the DEF is very small outside $100 \mathrm{~km}$ from the TC center, we have selected $r \leq 100 \mathrm{~km}$ and $z=1.5-13.2 \mathrm{~km}$ as the volume to compute the average dynamic efficiency of latent heat release denoted as $\tilde{\eta}_{H}$. Figure 12 indicates that the time series of $\tilde{\eta}_{H}$ remains in a range of $1.7 \%-1.8 \%$ prior to the RI onset from 0600 UTC 6 November, but increases to reach a maximum of $2.0 \%$ during the $24-\mathrm{h}$ RI period. The increase in $\tilde{\eta}_{H}$ is due to the increase of the baroclinicity and coincides with the increase in maximum wind speed in our simulation.

The system DEF, as a measure of the coupling of the convective heating and the DEF in the system, are calculated for the regions inside of $100-\mathrm{km}\left(\bar{\eta}_{\mathrm{H} 100}\right)$ and $1000-\mathrm{km}\left(\bar{\eta}_{\mathrm{H} 1000}\right)$ radii with (19). The $\bar{\eta}_{\mathrm{H} 1000}$ exhibits some variations between about 0.4 and $0.5 \%$ to 0600 UTC 7 November but increases rapidly during RI from a relative minimum of $0.35 \%$ at 0600 UTC to reach a peak of about $0.8 \%$ at 1800 UTC (Fig. 12b). On the other hand, the $\bar{\eta}_{\mathrm{H} 100}$ is always larger than $\bar{\eta}_{\mathrm{H} 1000}$. This suggests that $\mathrm{KE}$ conversion from convective heating occurred mainly in the inner-core region within a radius of $100 \mathrm{~km}$. The $\bar{\eta}_{\mathrm{H} 100}$ exhibits some variations between about 1.3 and $1.5 \%$ before the RI onset, except for an isolated peak of 
(a) $11 / 061800 \mathrm{UTC}$
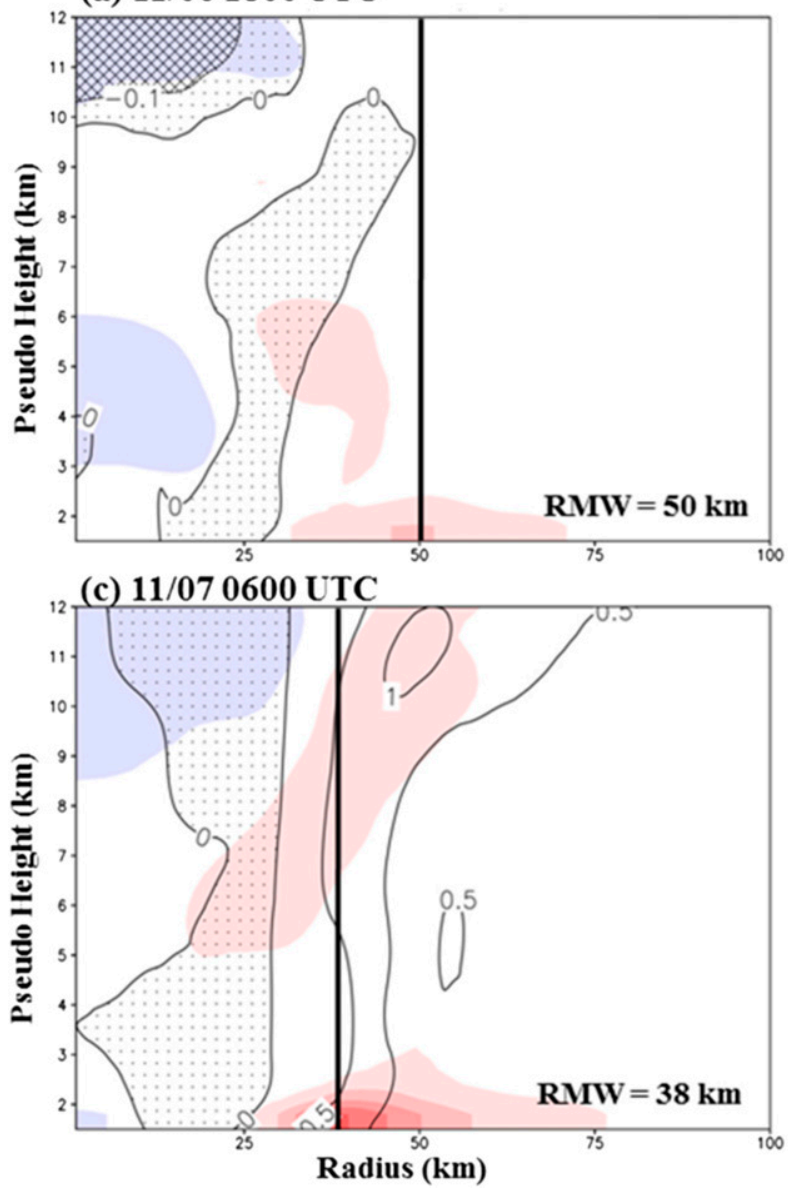

(b) 11/07 0000 UTC (RI onset)

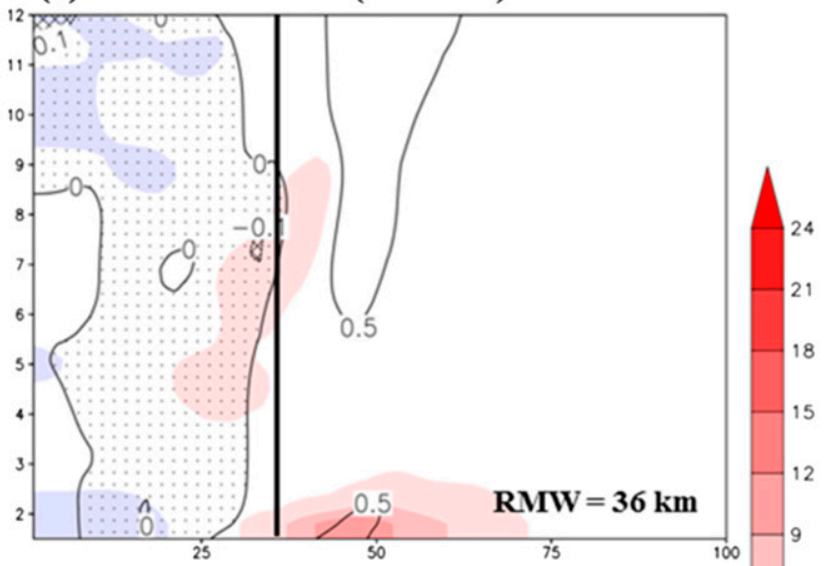

(d) 11/07 1800 UTC

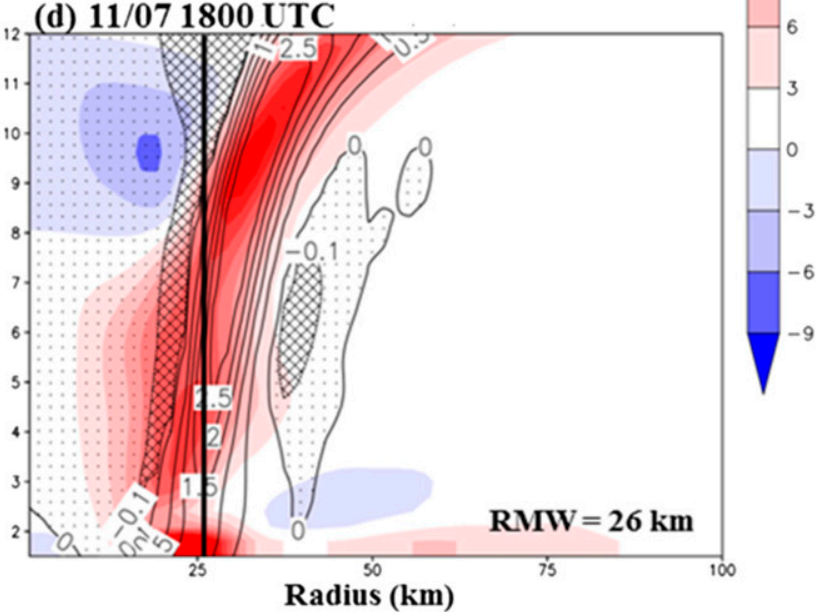

FIG. 9. As in Fig. 7, but showing the azimuthally averaged baroclinicity $\rho B\left(10^{-6} \mathrm{~s}^{-2}\right.$, color) and vertical velocity ( $\mathrm{m} \mathrm{s}^{-1}$, contour) in the $2-\mathrm{km}$ simulation. Dotted regions indicate subsidence, and hatched regions indicate strong downdraft $\left(<-0.1 \mathrm{~m} \mathrm{~s}^{-1}\right)$.

$1.7 \%$ at 1200 UTC 06 November. The peak reflects instantaneous convective heating $(\tilde{Q})$ at the same time, but the peak is not maintained. The $\bar{\eta}_{\mathrm{H} 100}$ increases at the RI onset, from $1.5 \%$ at 1800 UTC 6 November to about $2.0 \%$ at 0000 UTC 8 November (Fig. 12b). The conversion rate $C$, as computed with (17), doubled its value in the late stage of the RI period due to the increase of the convective heating (Fig. 12b).

Musgrave et al. (2012) presented a $K-V_{\max }$ diagram to analyze the life cycle of a TC. The $K-V_{\max }$ diagram is an elegant way to summarize the TC evolution with the volume-integrated $\mathrm{KE}$ inside a fixed radius and $V_{\max }$. We consider the volume integral of $\mathrm{KE}$ inside a radius of $100 \mathrm{~km}$ to focus on the inner-core region. Figure $12 \mathrm{c}$ indicates the $K-V_{\max }$ diagram from 1200 UTC 6 November (before the RI onset) to 0000 UTC 8 November (the end of the RI period). The volumeintegrated KE rapidly increases during the RI period although the volume-integrated KE still increases before the onset of RI. The rapid increase of the volume-integrated $\mathrm{KE}$ is consistent with the increase of the conversion term $\mathrm{C}$ inside of a radius of $100 \mathrm{~km}$ as seen in Fig. 12b. In addition, the increase of the volumeintegrated $\mathrm{KE}$ is well correlated with the increase of $V_{\max }$ during the RI period. The good correlation suggests that the DEF (the energy conversion to KE) can be a measurement of the intensity change of the TC vortex during RI.

The appearance of enhanced values of DEF during the RI period supports the notion of using the DEF to study the RI process. In particular, the increase of $\bar{\eta}_{\mathrm{H} 100}$ preceded the increase of $\bar{\eta}_{\mathrm{H} 1000}$ by $12 \mathrm{~h}$ and the increase may be linked to RI onset. The $\bar{\eta}_{\mathrm{H} 100}$, the system DEF within the radius of $100 \mathrm{~km}$, may be useful in measuring the vortex intensification before and after the onset of the RI.

Figure 13 summarizes the model results in radius-time Hovmöller diagram along with the model RMW at $1.5 \mathrm{~km}$ and the vortex intensity indicated. Weak and broad subsidence appeared within a radius of about $50 \mathrm{~km}$ before the RI onset (Fig. 13a). This subsidence 
(a) $\quad \mathrm{v}(\mathrm{m} / \mathrm{s}) \& Q\left(10^{\wedge} 3 \mathrm{k} / \mathrm{s}\right) 11 / 06 \quad 18$ UTC

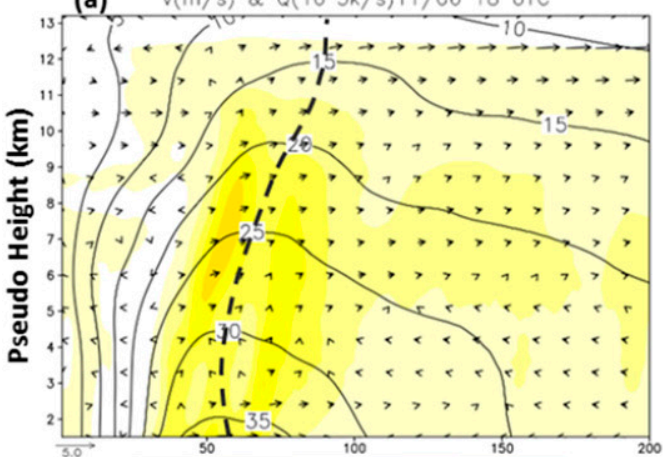

(c) $\mathrm{v}(\mathrm{m} / \mathrm{s}) \& Q\left(10^{\wedge} 3 \mathrm{k} / \mathrm{s}\right) 11 / 07,00$ UTC

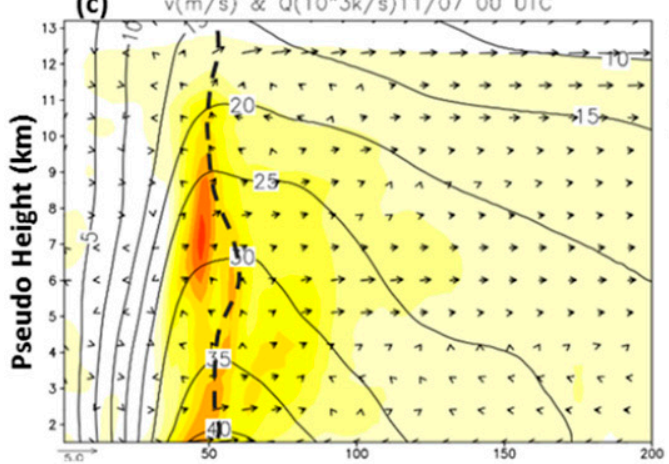

(e) $\mathrm{v}(\mathrm{m} / \mathrm{s}) \& Q\left(10^{\wedge} 3 \mathrm{k} / \mathrm{s}\right) 11 / 0706$ UTC
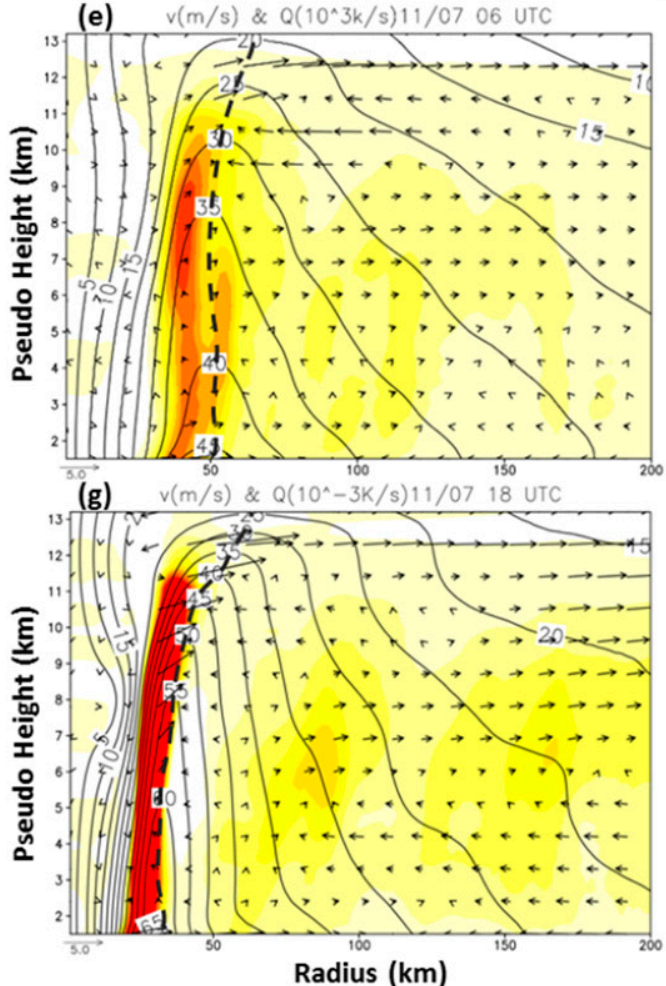

(b) $\quad$ eta $(\%) \& Q\left(10^{\wedge}-3 K / s\right) 11 / 0618$ UTC

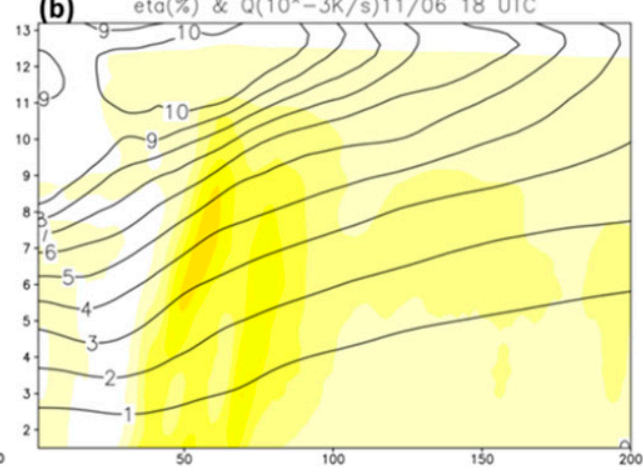

(d)

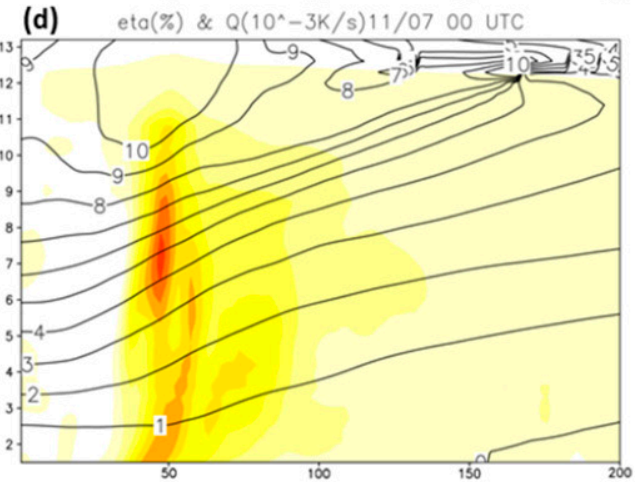

(f)

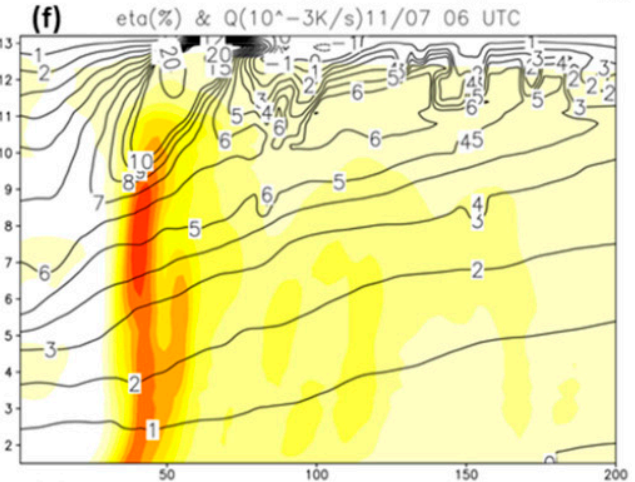

(h) $\quad \operatorname{eta}(\%) \& Q\left(10^{\wedge}-3 K / 5\right) 11 / 07 \quad 18$ UTC

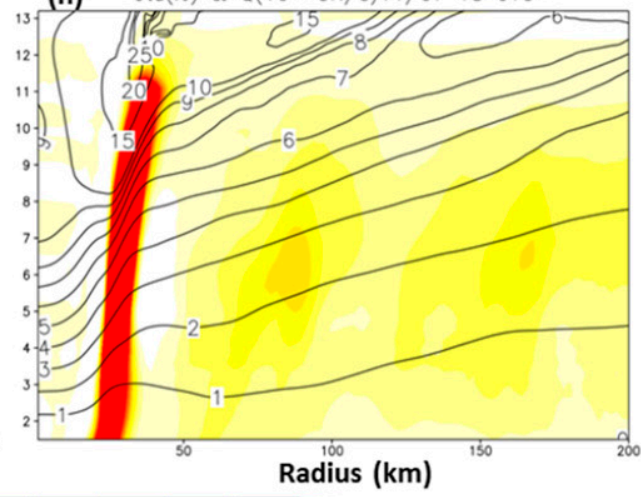

FIG. 10. Radius-height distribution of axisymmetric diabatic heating rate $Q\left(10^{-3} \mathrm{~K} \mathrm{~s}^{-1}\right.$, color, scale at bottom) and (a) tangential wind $v\left(\mathrm{~m} \mathrm{~s}^{-1}\right.$, isotachs, positive for cyclonic), radial wind $u$, and vertical velocity $w$ $\left(\mathrm{m} \mathrm{s}^{-1}\right.$, vectors, reference length at bottom left) on the section plane, and (b) conversion efficiency factor $\eta_{H}$ (\%, contours) at 1800 UTC 6 Nov 2013 in the 2-km simulation, for $r=0-200 \mathrm{~km}$ and $z=1.5-13.2 \mathrm{~km}$. (c)-(h) As in (a),(b), but at (c),(d) 0000, (e),(f) 0600, and (g),(h) 1800 UTC 7 Nov, respectively. All variables are temporally averaged over $6 \mathrm{~h}( \pm 3 \mathrm{~h}$ from the time indicated) from 5-min outputs, and the axes of maximum (axisymmetrical) tangential wind are depicted by thick dashed lines in the left panels. 
(a) $\mathrm{v}(\mathrm{m} / \mathrm{s}) \& \mathrm{Q}\left(10^{\wedge}-3 \mathrm{~K} / \mathrm{s}\right) 11 / 0618$ UTC

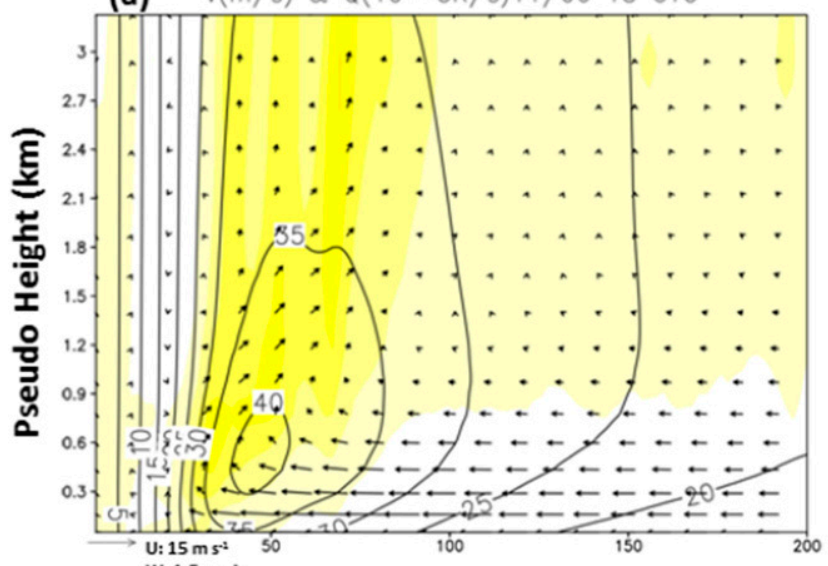

(c) $\underset{v}{\mathrm{~W}: 1.5 \mathrm{~ms}} \mathrm{~m} / \mathrm{s}) \& Q\left(10^{\wedge}-3 \mathrm{~K} / \mathrm{s}\right) 11 / 0706$ UTC

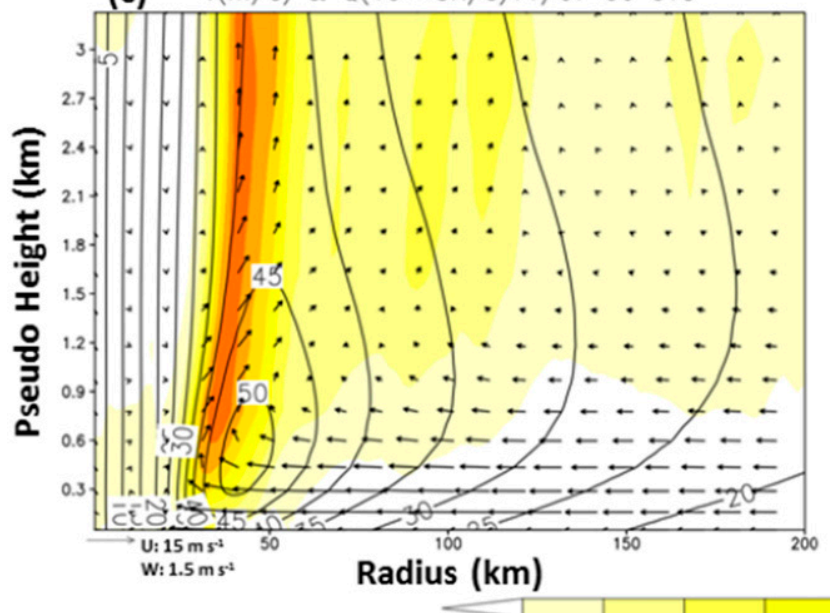

(b) $\quad \mathrm{v}(\mathrm{m} / \mathrm{s}) \& Q\left(10^{\wedge}-3 \mathrm{~K} / \mathrm{s}\right) 11 / 0700$ UTC

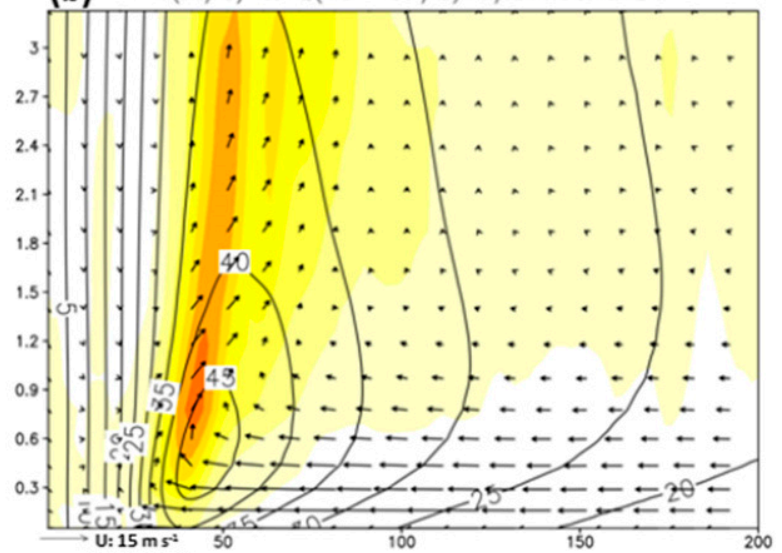

(d)

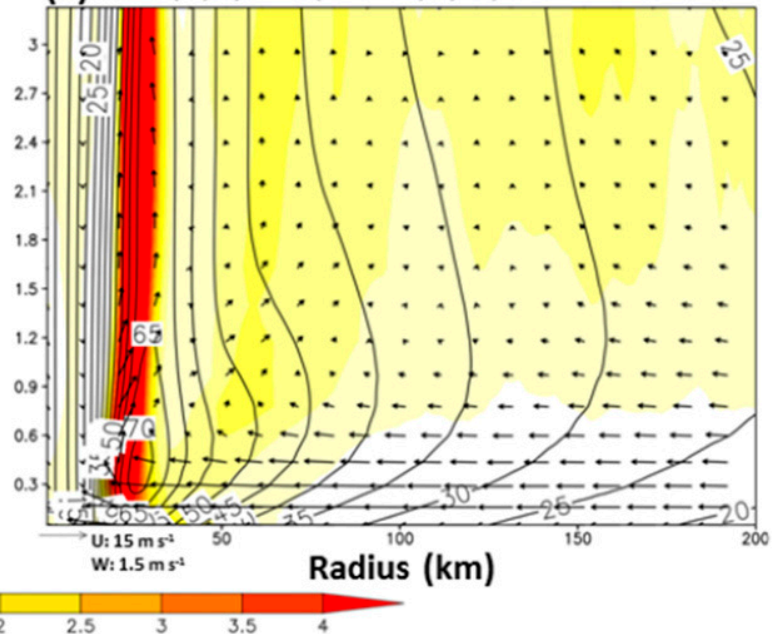

FIG. 11. As in Fig. 10a, but for the 6-h-averaged, axisymmetric distribution of $Q\left(10^{-3} \mathrm{~K} \mathrm{~s}^{-1}\right.$, color), $v$ ( $\mathrm{m} \mathrm{s}^{-1}$, isotachs), and $u$ and $w$ (m s${ }^{-1}$, vectors) below $z=3.3 \mathrm{~km}$ at (a) 1800 UTC 6 Nov, and (b) 0000, (c) 0600, and (d) 1800 UTC 7 Nov 2013, respectively.

was mainly associated with active convection around the model RMW (Fig. 13b). The azimuthally averaged updraft was still weak before the RI onset because the convection had strong asymmetry (Fig. 4a). The weak updraft in the free atmosphere is consistent with a relatively small change of the radial inflow $(\partial u / \partial r)$ in the $\mathrm{BL}$ in the $50-100-\mathrm{km}$ radius region (Figs. 11a and 13c). The baroclinicity was also weak so that the DEF was limited near the center (Figs. 13a and 13b). As a result, the convective heating between the $35-$ and $65-\mathrm{km}$ radius at 1200 UTC 6 November does not occur in the large DEF area and the increase of KE conversion is not maintained before the RI onset (Figs. 12b and 13b).

As the vortex intensified after the RI onset, the model RMW rapidly contracted, and the inertial stability and baroclinicity increased in the eye-core region (Figs. 8, 9, 13a, and 13d). During the RI period, the eyewall convection results in significant subsidence warming at the edge of the eye compared to the center of the eye (Fig. 13a). Figure 13a also indicates that eye warming enhances the baroclinicity near the eyewall and leads to expansion of the high DEF region. The high DEF region is collocated with the convective heating and the overall system DEF is large in the RI period (Fig. 13b).

Figure $13 \mathrm{c}$ shows that the radial inflow in the BL increased from 6 to $10 \mathrm{~m} \mathrm{~s}^{-1}$ in the $50-100-\mathrm{km}$ radius region. The increase of the unbalanced radial inflow can induce the $u(\partial u / \partial r)$ nonlinear effects and inward advection of the absolute angular momentum in the $\mathrm{BL}$ (Smith et al. 2009; Williams et al. 2013). As is also presented in Fig. 11, the inward advection of the absolute angular momentum, or the coupling of the inflow with the absolute vorticity, leads to the increase of the tangential wind and the contraction of the RMW in the BL (Fig. 13c). The nonlinear effect of $u(\partial u / \partial r)$ term may induce a shock-like structure with a very large $\partial u / \partial r$ in 

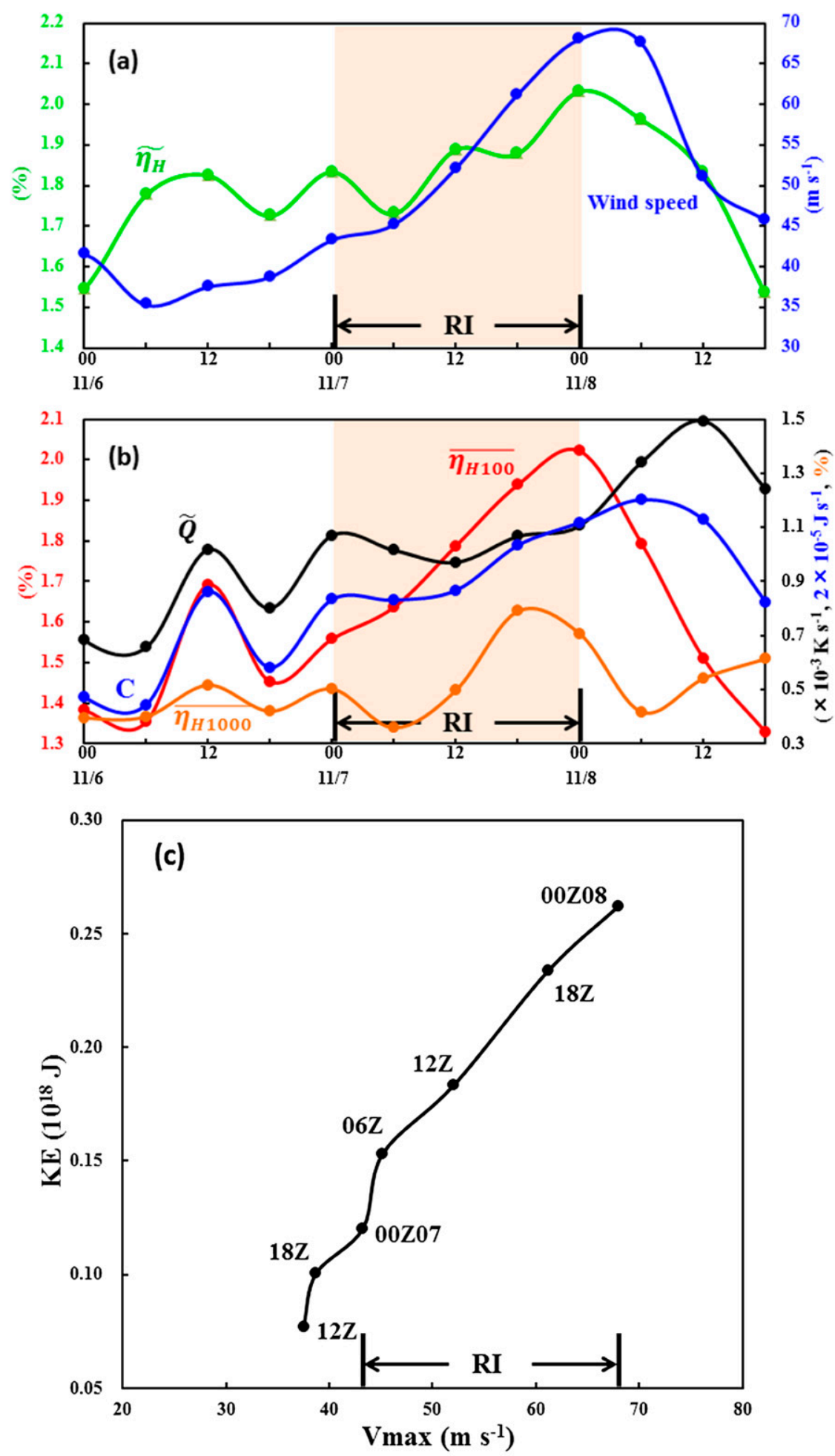

47

FIG. 12. Time series of (a) typhoon intensity ( $\mathrm{m} \mathrm{s}^{-1}$, blue, as in Fig. 1b) and mean conversion efficiency factor $\tilde{\eta}_{H}$ (\%, green), (b) overall DEF $\bar{\eta}_{\mathrm{H} 1000}\left(\%\right.$, brown), system DEF $\bar{\eta}_{\mathrm{H} 100}(\%$, red), system conversion rate $C\left(=\tilde{Q} \times \bar{\eta}_{\mathrm{H} 100} ; 2 \times 10^{-5} \mathrm{~J} \mathrm{~s}^{-1}\right.$, blue $)$, and mean heating rate $\tilde{Q}\left(10^{-3} \mathrm{~K} \mathrm{~s}^{-1}\right.$, black), and (c) integrated KE (ordinate; $\left.\times 10^{18} \mathrm{~J}\right)$ and the typhoon intensity (abscissa; $\mathrm{m} \mathrm{s}^{-1}$ ). The average area of $\tilde{\eta}_{H}, \bar{\eta}_{\mathrm{H} 100}$, and $\tilde{Q}$ and the integrated area of KE are inside 

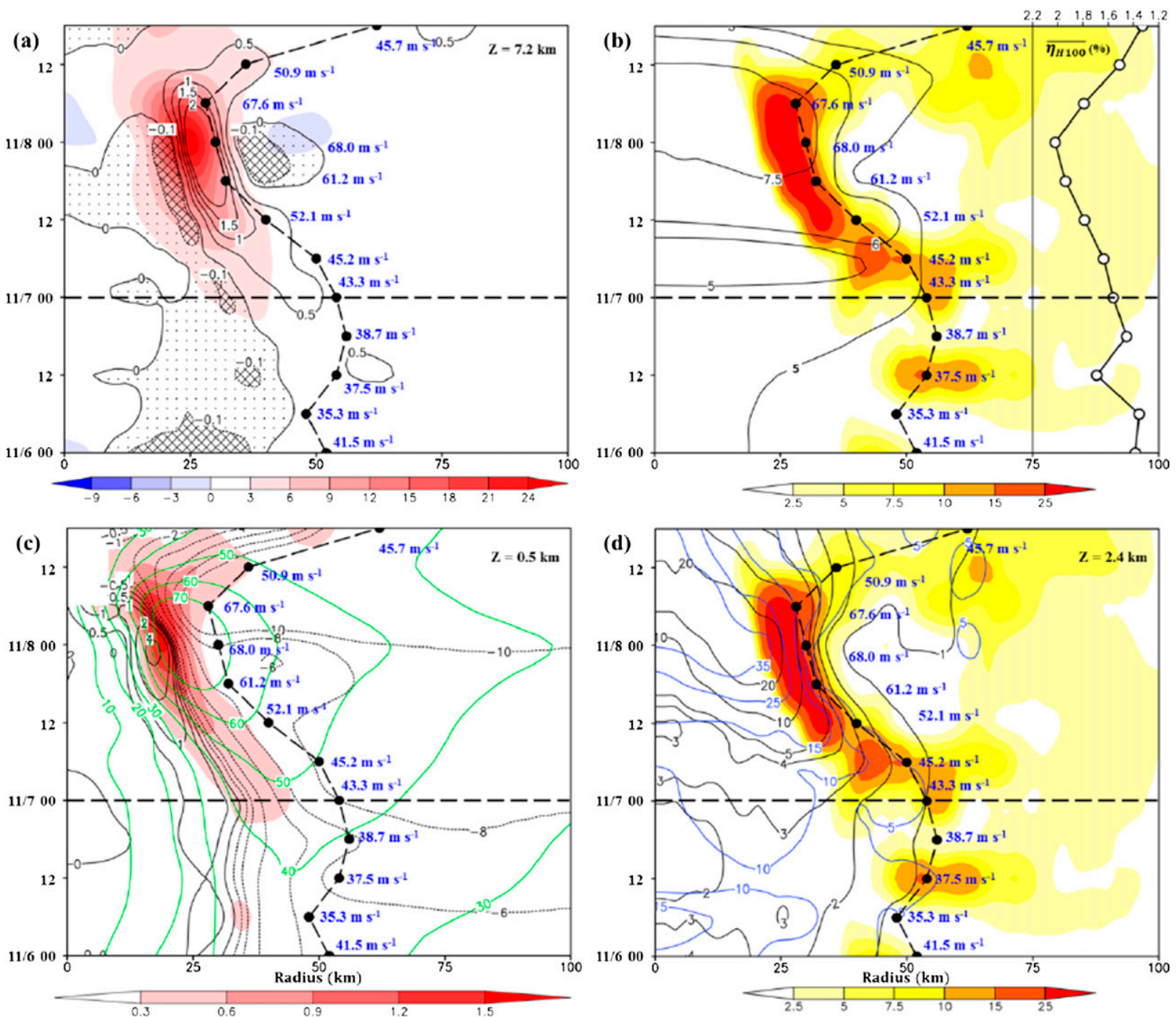

FIG. 13. Radius-time Hovmöller diagrams of (a) vertical velocity $\left(\mathrm{m} \mathrm{s}^{-1}\right.$, contour) and azimuthally averaged baroclinicity $\rho B\left(10^{-6} \mathrm{~s}^{-2}\right.$, color) at $7.2 \mathrm{~km},(\mathrm{~b})$ mean diabatic heating rate $Q\left(\mathrm{~K} \mathrm{~h}^{-1}\right.$, color $)$ and $\eta_{H}(\%$, contour $)$ over $z=1.5-12 \mathrm{~km}$ within $\pm 3 \mathrm{~h}$, (c) radial wind (10, $8,6, \pm 4, \pm 2, \pm 1, \pm 0.5 \mathrm{~m} \mathrm{~s}^{-1}$, black contour), tangential wind $\left(\mathrm{m} \mathrm{s}^{-1}\right.$, green contour), and vertical velocity ( $\mathrm{m} \mathrm{s}^{-1}$, color) at $0.5 \mathrm{~km}$, and (d) azimuthally averaged inertial stability $\rho C\left(10^{-6} \mathrm{~s}^{-2}\right.$, black contour), PV (PVU, blue contour) at $2.4 \mathrm{~km}$, and mean diabatic heating rate $Q\left(\mathrm{~K} \mathrm{~h}^{-1}\right.$, color $)$. In all panels, the dashed lines with black points denote the mean RMW $(\mathrm{km})$ at $1.5 \mathrm{~km}$ within $\pm 3 \mathrm{~h}$, and the horizontal dashed lines mark the onset time of RI. The values with blue color denote the peak surface wind at each time (corresponding to the blue line in Fig. 1b). In panel (b), the black solid line with open circles marks $\bar{\eta}_{\mathrm{H} 100}(\%)$. The dotted regions and hatched regions indicate subsidence and strong downdraft $\left(<-0.1 \mathrm{~m} \mathrm{~s}^{-1}\right)$.

the $\mathrm{BL}$ around radii of $30-40 \mathrm{~km}$ (Fig. 13c). The shocklike structure is linked to the formation of a strong eyewall updraft on the inner edge of the maximum radial inflow. Note that the maximum updraft in the BL is located inside the RMW (Fig. 13c). Thus, the eyewall deep convection is coupled with a large PV field in the large inertial stability region inside the RMW during the RI period (Figs. 8d, 9d, and 13d). This is in agreement

$r=100 \mathrm{~km}$ and $z=1.5-13.2 \mathrm{~km}$, while $\tilde{\eta}_{H}, \bar{\eta}_{\mathrm{H} 100}, \tilde{Q}$, and $\bar{\eta}_{\mathrm{H} 1000}$ are also temporally averaged (over $6 \mathrm{~h}, \pm 3 \mathrm{~h}$ from the time indicated). The shaded areas depict the RI period. In panel (c), the time series are limited before the RI onset (1200 UTC 6 Nov) to the end of the RI period (0000 UTC 8 Nov). 
with the finding of many of the previous studies (e.g., Schubert and Hack 1982; Hausman et al. 2006; Smith and Montgomery 2016), that the convective heating occurred in regions of high inertial stability or convective heating coupled with high PV field can efficiently intensify the $V_{\max }$. These results highlight the importance of the BL radial inflow in organizing the eyewall updraft and the deep convection inside the RMW.

\section{Conclusions}

There are many factors across a wide range of scales that affect the RI process. These factors include largescale environmental conditions, mesoscale vortex structure, and the convective-scale diabatic heating. Since RI is more likely to occur in an intense TC (Shu et al. 2012), it is possible that nonlinear interaction across scales is important in the RI process. Hendricks et al. (2010) showed that once a favorable large-scale environment is in place to allow the TCs to intensify, there is no further distinction in synoptic conditions on whether RI will occur or not. On the other hand, factors such as the degree of symmetry and the deep convection in the inertially stable region may be important for internal dynamics of RI (e.g., Schubert and Hack 1982; Smith and Montgomery 2016). The PV mixing and axisymmetrization dynamics (Schubert et al. 1999) and the production of a PV convective tower (Hausman et al. 2006) near the eyewall may be essential for the RI processes. The large unbalanced radial inflow in the BL may also exert a strong control on the location of the eyewall updraft and organization of deep convection (Montgomery and Smith 2017). Bell et al. (2018) and Martinez et al. (2019, manuscript submitted to J. Atmos. Sci.) indicate the importance of PV mixing and the presence of high PV convective tower in Hurricane Patricia (2015) during the RI period.

As the most intense tropical cyclone in the western hemisphere, Supertyphoon Haiyan had an RI period in which the maximum wind speed increased by $31 \mathrm{~m} \mathrm{~s}^{-1}$ in $24 \mathrm{~h}$ over the warm ocean and in a low vertical wind shear environment. We have employed a $2-\mathrm{km}$ horizontal resolution full-physics cloud-resolving model to simulate the RI of Haiyan. Our simulation is in general agreement with the observations of Haiyan's structure. We have introduced the energy diagnostic DEF, which measures the effectiveness of convective heating in producing KE. DEF is large in the presence of eye warming and strong baroclinicity in the eye region (HS86). We have investigated the internal dynamics of RI using this DEF diagnostic.

Our results highlight axisymmetrization prior to the onset of the RI period and the nonlinear feedback process to collocate the large DEF area with strong convection near the eyewall during the RI period. The TC becomes more symmetric prior to and during the RI period, possibly due to PV mixing in the lower atmosphere. In the presence of increased vortex intensity and the inertial stability in the vortex core, the eyewall convection results in significant subsidence warming at the edge of the eye rather than at the center of the eye. The overall eye warming enhances the baroclinicity near the eyewall and increases the DEF value in the eye region. The collocation of the deep convection and the large DEF area thus increases $\mathrm{KE}$ and $V_{\max }$ significantly. The system DEF within a $100-\mathrm{km}$ radius is increased by $33 \%$ in $12 \mathrm{~h}$ during the RI period. The rapid vortex intensity increase also causes an increase of unbalanced radial inflow in the BL. This inflow, coupled with large absolute vorticity, also leads to an increase of the tangential wind within the BL near the eyewall and to the contraction of the RMW. The large unbalanced BL radial inflow with the nonlinear effect of $u(\partial u / \partial r)$ exerts a strong control on the organization of deep convection. The BL radial inflow helps to form an eyewall updraft and deep convection in regions of high inertial stability inside the RMW. The deep convection and the PV become locked together to further efficiently intensify the TC.

Apparently, the PV mixing and symmetrization, the eyewall deep convection, the subsidence in the eye, the BL unbalanced radial flow, the contraction of the RMW, the coupling of deep convection with high PV, and the interaction of eyewall convection with the TC vortex, are all indispensable components in the nonlinear process of RI. Our simulation emphasizes the importance of using a model resolution of at least $2 \mathrm{~km}$ to simulate the internal nonlinear processes of RI. While the unbalanced dynamics in the BL is a crucial component of RI, the DEF diagnosis suggests the usefulness of the balanced dynamics in diagnosing the process of RI. The DEF analysis may diagnose well the RI processes, which involve the interaction of convection with balanced and unbalanced dynamics. In particular, the system DEF within $100-\mathrm{km}$ radius region can be a useful parameter to understand RI and to improve RI forecasts.

Acknowledgments. The authors thank three anonymous reviewers, Dr. W. H. Schubert, Dr. M. S. Peng, and P. Ciesielski for their valuable and constructive comments. This study was supported by the Office of Naval Research Global under Grant N62909-15-1-2008, and by the Ministry of Science and Technology (MOST) of Taiwan under Grants MOST-107-2628-M-002-016, MOST-106-2119-M-002-016, MOST-104-2111-M-002-002MY3, and MOST-105-2111-M-003-003-MY3. The 
numerical experiments were performed using the Earth Simulator at the Japan Agency for Marine-Earth Science and Technology (JAMSTEC). The National Center for High-performance Computing (NCHC) of Taiwan is also acknowledged for additional resources to calculate and the diagnostic model. The satellite images in Fig. 2 are provided by the Naval Research Laboratory (NRL).

\section{REFERENCES}

Akter, N., and K. Tsuboki, 2012: Numerical simulation of Cyclone Sidr using a cloud-resolving model: Characteristics and formation process of an outer rainband. Mon. Wea. Rev., 140, 789-810, https://doi.org/10.1175/2011MWR3643.1.

Alvey, G. R., III, J. Zawislak, and E. Zipser, 2015: Precipitation properties observed during tropical cyclone intensity change. Mon. Wea. Rev., 143, 4476-4492, https://doi.org/10.1175/ MWR-D-15-0065.1.

Bell, M. M., J. Martinez, J. D. Doyle, and R. F. Rogers, 2018: Potential vorticity structure and evolution of Hurricane Patricia (2015). 33rd Conf. on Hurricanes and Tropical Meteorology, Ponte Vedra, FL, Amer. Meteor. Soc., 12B.3, https://ams.confex. com/ams/33HURRICANE/webprogram/Paper340245.html.

Bister, M., and K. A. Emanuel, 1998: Dissipative heating and hurricane intensity. Meteor. Atmos. Phys., 65, 233-240, https:// doi.org/10.1007/BF01030791.

Braun, S. A., 2002: A cloud-resolving simulation of Hurricane Bob (1991): Storm structure and eyewall buoyancy. Mon. Wea. Rev., 130, 1573-1592, https://doi.org/10.1175/1520-0493(2002) $130<1573$ :ACRSOH $>2.0 . \mathrm{CO} ; 2$.

Bui, H. H., R. K. Smith, M. T. Montgomery, and J. Peng, 2009: Balanced and unbalanced aspects of tropical cyclone intensification. Quart. J. Roy. Meteor. Soc., 135, 1715-1731, https://doi.org/10.1002/qj.502.

DeMaria, M., J. A. Knaff, and C. Sampson, 2007: Evaluation of longterm trends in tropical cyclone intensity forecasts. Meteor. Atmos. Phys., 97, 19-28, https://doi.org/10.1007/s00703-006-0241-4.

_ C. C. R. Sampson, J. A. Knaff, and K. D. Musgrave, 2014: Is tropical cyclone intensity guidance improving? Bull. Amer. Meteor. Soc., 95, 387-398, https://doi.org/10.1175/BAMS-D12-00240.1.

Eliassen, A., 1952: Slow thermally or frictionally controlled meridional circulation in a circular vortex. Astrophys. Norv., 5, $60 \mathrm{pp}$.

Elsberry, R. L., T. D. B. Lambert, and M. A. Boothe, 2007: Accuracy of Atlantic and eastern North Pacific tropical cyclone intensity forecast guidance. Wea. Forecasting, 22, 747-762, https://doi.org/10.1175/WAF1015.1.

Guimond, S. R., G. M. Heymsfield, and F. J. Turk, 2010: Multiscale observations of Hurricane Dennis (2005): The effects of hot towers on rapid intensification. J. Atmos. Sci., 67, 633-654, https://doi.org/10.1175/2009JAS3119.1.

$\longrightarrow,-$, P. D. Reasor, and A. C. Didlake Jr., 2016: The rapid intensification of Hurricane Karl (2010): New remote sensing observations of convective bursts from the Global Hawk platform. J. Atmos. Sci., 73, 3617-3639, https://doi.org/10.1175/ JAS-D-16-0026.1.

Hack, J. J., and W. H. Schubert, 1986: Nonlinear response of atmospheric vortices to heating by organized cumulus convection. J. Atmos. Sci., 43, 1559-1573, https://doi.org/10.1175/ 1520-0469(1986)043<1559:NROAVT>2.0.CO;2.
Harnos, D. S., and S. W. Nesbitt, 2011: Convective structure in rapidly intensifying tropical cyclones as depicted by passive microwave measurements. Geophys. Res. Lett., 38, L07805, https://doi.org/10.1029/2011GL047010.

- and _ 2016a: Passive microwave quantification of tropical cyclone inner-core cloud populations relative to subsequent intensity change. Mon. Wea. Rev., 144, 4461-4482, https:// doi.org/10.1175/MWR-D-15-0090.1.

$\longrightarrow$, and — 2016b: Varied pathways for simulated tropical cyclone rapid intensification. Part II: Vertical motion and cloud populations. Quart. J. Roy. Meteor. Soc., 142, 1832-1846, https://doi.org/10.1002/qj.2778.

Hausman, S. A., K. V. Ooyama, and W. H. Schubert, 2006: Potential vorticity structure of simulated hurricanes. J. Atmos. Sci., 63, 87-108, https://doi.org/10.1175/JAS3601.1.

Hendricks, E. A., W. H. Schubert, R. K. Taft, H. Wang, and J. P. Kossin, 2009: Life cycles of hurricane-like vorticity rings. J. Atmos. Sci., 66, 705-722, https://doi.org/10.1175/2008JAS2820.1.

- - M. S. Peng, B. Fu, and T. Li, 2010: Quantifying environmental control on tropical cyclone intensity change. Mon. Wea. Rev., 138, 3243-3271, https://doi.org/10.1175/ 2010MWR3185.1.

Ito, K., 2016: Errors in tropical cyclone intensity forecast by RSMC Tokyo and statistical correction using environmental parameters. SOLA, 12, 247-252.

Kadowaki, T., and K. Yoshimoto, 2012: A new inner model with a higher horizontal resolution (TL319) in JMA's Global 4D-Var data assimilation system. CAS/JSC WGNE Res. Activ. Atmos. Oceanic Modell., 42, 01.09-01.10.

Kanada, S., T. Takemi, M. Kato, S. Yamasaki, H. Fudeyasu, K. Tsuboki, O. Arakawa, and I. Takayabu, 2017: A multimodel intercomparison of an intense typhoon in future, warmer climates by four $5-\mathrm{km}$-mesh models. J. Climate, 30, 6017-6036, https://doi.org/10.1175/JCLI-D-16-0715.1.

Kaplan, J., and M. DeMaria, 2003: Large-scale characteristics of rapidly intensifying tropical cyclones in the North Atlantic basin. Wea. Forecasting, 18, 1093-1108, https://doi.org/10.1175/ 1520-0434(2003)018<1093:LCORIT>2.0.CO;2.

Kieper, M. E., and H. Jiang, 2012: Predicting tropical cyclone rapid intensification using the $37 \mathrm{GHz}$ ring pattern identified from passive microwave measurements. Geophys. Res. Lett., 39, L13804, https://doi.org/10.1029/2012GL052115.

Kossin, J. P., and W. H. Schubert, 2001: Mesovortices, polygonal flow patterns, and rapid pressure falls in hurricane-like vortices. J. Atmos. Sci., 58, 2196-2209, https://doi.org/10.1175/15200469(2001)058<2196:MPFPAR >2.0.CO;2.

_- and —_, 2004: Mesovortices in Hurricane Isabel, 2004. Bull. Amer. Meteor. Soc., 85, 151-153, https://doi.org/10.1175/ BAMS-85-2-151.

Kuo, H.-C., W.-Y. Cheng, Y.-T. Yang, E. A. Hendricks, and M. S. Peng, 2016: Deep convection in elliptical and polygonal eyewalls of tropical cyclones. J. Geophys. Res. Atmos., 121, 14 456-14 468, https://doi.org/10.1002/2016JD025317.

Lin, I-I, I. F. Pun, and C. C. Lien, 2014: "Category-6" Supertyphoon Haiyan in global warming hiatus: Contribution from subsurface ocean warming. Geophys. Res. Lett., 41, 8547-8553, https://doi.org/10.1002/2014GL061281.

Liu, A. Q., G. W. K. Moore, K. Tsuboki, and I. A. Renfrew, 2004: A high-resolution simulation of convective roll clouds during a cold-air outbreak. Geophys. Res. Lett., 31, L03101, https:// doi.org/10.1029/2003GL018530.

Marks, F. D., and L. K. Shay, 1998: Landfalling tropical cyclones: Forecast problems and associated research opportunities. 
Bull. Amer. Meteor. Soc., 79, 305-323, https://doi.org/10.1175/ 1520-0477(1998)079<0305:LTCFPA > 2.0.CO;2.

Miyamoto, Y., and T. Takemi, 2013: A transition mechanism for the axisymmetric spontaneous intensification of tropical cyclones. J. Atmos. Sci., 70, 112-129, https://doi.org/10.1175/ JAS-D-11-0285.1.

$\longrightarrow$, and - , 2015: A triggering mechanism for rapid intensification of tropical cyclones. J. Atmos. Sci., 72, 2666-2681, https://doi.org/10.1175/JAS-D-14-0193.1.

Montgomery, M. T., and R. K. Smith, 2017: Recent developments in the fluid dynamics of tropical cyclones. Annu. Rev. Fluid Mech., 49, 541-574, https://doi.org/10.1146/annurev-fluid010816-060022.

—, M. E. Nicholls, T. A. Cram, and A. B. Saunders, 2006: A vortical hot tower route to tropical cyclogenesis. J. Atmos. Sci., 63, 355-386, https://doi.org/10.1175/JAS3604.1.

Musgrave, K. D., R. K. Taft, J. L. Vigh, B. D. McNoldy, and W. H. Schubert, 2012: Time evolution of the intensity and size of tropical cyclones. J. Adv. Model. Earth Syst., 4, M08001, https://doi.org/10.1029/2011MS000104.

Nolan, D. S., Y. Moon, and D. P. Stern, 2007: Tropical cyclone intensification from asymmetric convection: Energetics and efficiency. J. Atmos. Sci., 64, 3377-3405, https://doi.org/ 10.1175/JAS3988.1.

Nomura, M., and K. Tsuboki, 2012: Numerical study of precipitation intensification and ice-phase microphysical processes in typhoon spiral band. J. Meteor. Soc. Japan, 90, 685-699, https://doi.org/10.2151/jmsj.2012-508.

Paull, G., K. Menelaou, and M. K. Yau, 2017: Sensitivity of tropical cyclone intensification to axisymmetric heat sources: The role of inertial stability. J. Atmos. Sci., 74, 2325-2340, https:// doi.org/10.1175/JAS-D-16-0298.1.

Pendergrass, A. G., and H. E. Willoughby, 2009: Diabatically induced secondary flows in tropical cyclones. Part I: Quasisteady forcing. Mon. Wea. Rev., 137, 805-821, https://doi.org/ 10.1175/2008MWR2657.1.

Rafiuddin, M., H. Uyeda, and M. Kato, 2013: Development of an arc-shaped precipitation system during the pre-monsoon period in Bangladesh. Meteor. Atmos. Phys., 120, 165-176, https://doi.org/10.1007/s00703-013-0244-x.

Rogers, R., and Coauthors, 2013a: NOAA's Hurricane Intensity Forecasting Experiment: A progress report. Bull. Amer. Meteor. Soc., 94, 859-882, https://doi.org/10.1175/BAMS-D-12-00089.1.

- P. Reasor, and S. Lorsolo, 2013b: Airborne Doppler observations of the inner-core structural differences between intensifying and steady-state tropical cyclones. Mon. Wea. Rev., 141, 2970-2991, https://doi.org/10.1175/MWR-D-12-00357.1.

_ J. A. Zhang, J. Zawislak, H. Jiang, G. R. Alvey III, E. J. Zipser, and S. N. Stevenson, 2016: Observations of the structure and evolution of Hurricane Edouard (2014) during intensity change. Part II: Kinematic structure and the distribution of deep convection. Mon. Wea. Rev., 144, 3355-3376, https:// doi.org/10.1175/MWR-D-16-0017.1.

Schubert, W. H., and J. J. Hack, 1982: Inertial stability and tropical cyclone development. J. Atmos. Sci., 39, 1687-1697, https:// doi.org/10.1175/1520-0469(1982)039<1687:ISATCD>2.0.CO;2.

—, M. T. Montgomery, R. K. Taft, T. A. Guinn, S. R. Fulton, J. P. Kossin, and J. P. Edwards, 1999: Polygonal eyewalls, asymmetric eye contraction, and potential vorticity mixing in Hurricanes. J. Atmos. Sci., 56, 1197-1223, https://doi.org/10.1175/15200469(1999)056<1197:PEAECA > 2.0.CO;2.

—, C. M. Rozoff, J. L. Vigh, B. D. McNoldy, and J. P. Kossin, 2007: On the distribution of subsidence in the hurricane eye.
Quart. J. Roy. Meteor. Soc., 133, 595-605, https://doi.org/ 10.1002/qj.49.

Shapiro, L. J., and H. Willoughby, 1982: The response of balanced hurricanes to local sources of heat and momentum. J. Atmos. Sci., 39, 378-394, https://doi.org/10.1175/1520-0469(1982) 039<0378:TROBHT $>2.0$. CO;2.

Shimizu, S., H. Uyeda, Q. Moteki, T. Maesaka, Y. Takaya, K. Akaeda, T. Kato, and M. Yoshizaki, 2008: Structure and formation mechanism on the 24 May 2000 supercell-like storm developing in a moist environment over the Kanto Plain, Japan. Mon. Wea. Rev., 136, 2389-2407, https://doi.org/10.1175/ 2007MWR2155.1.

Shu, S., and F. Zhang, 2015: Influence of equatorial waves on the genesis of Super Typhoon Haiyan (2013). J. Atmos. Sci., 72 4591-4613, https://doi.org/10.1175/JAS-D-15-0016.1.

_ probability of rapidly intensifying tropical cyclones in the western North Pacific basin. Wea. Forecasting, 27, 411-423, https://doi.org/10.1175/WAF-D-11-00042.1.

Smith, R. K., and M. T. Montgomery, 2016: The efficiency of diabatic heating and tropical cyclone intensification. Quart. J. Roy. Meteor. Soc., 142, 2081-2086, https://doi.org/10.1002/qj.2804. , and V. S. Nguyen, 2009: Tropical cyclone spin-up revisited. Quart. J. Roy. Meteor. Soc., 135, 1321-1335, https:// doi.org/10.1002/qj.428.

Sun, N., and F. Weng, 2008: Evaluation of Special Sensor Microwave Imager/Sounder (SSMIS) environmental data records. IEEE Trans. Geosci. Remote Sens., 46, 1006-1016, https:// doi.org/10.1109/TGRS.2008.917368.

Tao, C., and H. Jiang, 2015: Distributions of shallow to very deep precipitation-convection in rapidly intensifying tropical cyclones. J. Climate, 28, 8791-8824, https://doi.org/10.1175/JCLID-14-00448.1.

- - , and J. Zawislak, 2017: The relative importance of stratiform and convective rainfall in rapidly intensifying tropical cyclones. Mon. Wea. Rev., 145, 795-809, https:// doi.org/10.1175/MWR-D-16-0316.1.

Tsuboki, K., 2008: High-resolution simulations of high-impact weather systems using the cloud-resolving model on the Earth Simulator. High Resolution Numerical Modeling of the Atmosphere and Ocean, K. Hamilton and W. Ohfuchi, Eds., Springer, 141-156.

_- and A. Sakakibara, 2002: Large-scale parallel computing of cloud resolving storm simulator. High Performance Computing, H. P. Zima et al., Eds., Springer, 243-259.

- , and —, 2007: Numerical Prediction of High-Impact Weather Systems: The Textbook for the Seventeenth IHP Training Course in 2007. Hydrospheric Atmospheric Research Center, Nagoya University, and UNESCO, 273 pp.

- M. K. Yoshioka, T. Shinoda, M. Kato, S. Kanada, and A. Kitoh, 2015: Future increase of supertyphoon intensity associated with climate change. Geophys. Res. Lett., 42, 646652, https://doi.org/10.1002/2014GL061793.

Tsujino, S., K. Tsuboki, and H.-C. Kuo, 2017: Structure and maintenance mechanism of long-lived concentric eyewalls associated with simulated Typhoon Bolaven (2012). J. Atmos. Sci., 74, 3609-3634, https://doi.org/10.1175/JAS-D-16-0236.1.

Vigh, J. L., and W. H. Schubert, 2009: Rapid development of the tropical cyclone warm core. J. Atmos. Sci., 66, 3335-3350, https://doi.org/10.1175/2009JAS3092.1.

Wang, C.-C., G. T.-J. Chen, and S.-Y. Huang, 2011: Remote trigger of deep convection by cold outflow over the Taiwan Strait in the mei-yu season: A modeling study of the 8 June 2007 
case. Mon. Wea. Rev., 139, 2854-2875, https://doi.org/10.1175/ 2011MWR3613.1.

, H.-C. Kuo, Y.-H. Chen, H.-L. Huang, C.-H. Chung, and K. Tsuboki, 2012: Effects of asymmetric latent heating on typhoon movement crossing Taiwan: The case of Morakot (2009) with extreme rainfall. J. Atmos. Sci., 69, 3172-3196, https://doi.org/10.1175/JAS-D-11-0346.1.

, Y.-H. Chen, H.-C. Kuo, and S.-Y. Huang, 2013: Sensitivity of typhoon track to asymmetric latent heating/rainfall induced by Taiwan topography: A numerical study of Typhoon Fanapi (2010). J. Geophys. Res., 118, 3292-3308, https://doi.org/10.1002/ jgrd.50351.

- J. C.-S. Hsu, G. T.-J. Chen, and D.-I. Lee, 2014: A study of two propagating heavy-rainfall episodes near Taiwan during SoWMEX/TiMREX IOP-8 in June 2008. Part II: Sensitivity tests on the roles of synoptic conditions and topographic effects. Mon. Wea. Rev., 142, 2644-2664, https://doi.org/10.1175/ MWR-D-13-00330.1.

_, B.-X. Lin, C.-T. Chen, and S.-H. Lo, 2015a: Quantifying the effects of long-term climate change on tropical cyclone rainfall using cloud-resolving models: Examples of two landfall typhoons in Taiwan. J. Climate, 28, 66-85, https://doi.org/10.1175/ JCLI-D-14-00044.1.

, H.-C. Kuo, R. H. Johnson, C.-Y. Lee, S.-Y. Huang, and Y.-H. Chen, 2015b: A numerical study of convection in rainbands of Typhoon Morakot (2009) with extreme rainfall: Roles of pressure perturbations with low-level wind maxima. Atmos. Chem. Phys., 15, 11 097-11 115, https://doi.org/10.5194/acp-1511097-2015.

—, B.-K. Chiou, G. T.-J. Chen, H.-C. Kuo, and C.-H. Liu, 2016: A numerical study of back-building process in a quasistationary rainband with extreme rainfall over northern Taiwan during 11-12 June 2012. Atmos. Chem. Phys., 16, 1235912 382, https://doi.org/10.5194/acp-16-12359-2016.

Wang, Y., and C.-C. Wu, 2004: Current understanding of tropical cyclone structure and intensity changes-A review. Meteor. Atmos. Phys., 87, 257-278, https://doi.org/10.1007/s00703-0030055-6.

Williams, G. J., R. K. Taft, B. D. McNoldy, and W. H. Schubert, 2013: Shock-like structures in the tropical cyclone boundary layer. J. Adv. Model. Earth Syst., 5, 338-353, https://doi.org/ 10.1002/jame.20028.

Zagrodnik, J. P., and H. Jiang, 2014: Rainfall, convection, and latent heating distributions in rapidly intensifying tropical cyclones. J. Atmos. Sci., 71, 2789-2809, https://doi.org/10.1175/ JAS-D-13-0314.1.

Zawislak, J., H. Jiang, G. R. Alvey III, E. J. Zipser, R. F. Rogers, J. A. Zhang, and S. N. Stevenson, 2016: Observations of the structure and evolution of Hurricane Edouard (2014) during intensity change. Part I: Relationship between the thermodynamic structure and precipitation. Mon. Wea. Rev., 144, 3333-3354, https://doi.org/10.1175/MWR-D-16-0018.1. 